\title{
Der Bevölkerungswandel in Deutschland und seine Auswirkungen auf die sozialen Sicherungssysteme
}

Umlagefinanzierte Systeme wie die deutsche Kranken- und die Rentenversicherung hängen in ihrer Funktionsfähigkeit entscheidend von dem Verhältnis zwischen den Beitragszahlern und den Leistungsbeziehern im jeweiligen Teilsystem sozialer Sicherung ab. ${ }^{1}$ Aufgrund der Bevölkerungsentwicklung in Deutschland, die gekennzeichnet ist durch einen zahlenmäßigen Rückgang der Bevölkerung bei gleichzeitiger Erhöhung des Anteils älterer Personen an der Gesamtbevölkerung, verschlechtert sich dieses Verhältnis zunehmend. Dies ist keine spekulative Prognose mit Unsicherheitsfaktoren, denn die Demographie hat einen weiten Blick in die Zukunft: Die potentiellen Leistungsbezieher, aber auch die Eltern etwa des Jahres 2025 existieren bereits. Der vorliegende Beitrag schildert die relevanten Daten, diskutiert ihre negativen Folgen auf die beiden wichtigsten Teilsysteme sozialer Sicherung und skizziert Ansätze zur Lösung der häufig als solcher bezeichneten Demographiefalle. ${ }^{2}$

\section{Peter Kostorz und Friedrich E. Schnapp}

\section{Der empirische Befund}

Unter Bevölkerung versteht man im Allgemeinen die Gesamtzahl der Einwohner innerhalb eines politisch abgegrenzten Gebietes, ${ }^{3}$ unter demographischem Wandel entsprechend die Summe jener Prozesse, die die $\mathrm{Zu}-$ sammensetzung und Zahl der Einwohner beeinflussen ${ }^{4}$. Die bisherige Entwicklung der bundesdeutschen Bevölkerung ist hinsichtlich ihrer Altersstruktur bekannt: Die Pyramide von damals wurde zur „zerzausten Wettertanne ${ }^{\text {“5 }}$ von heute (Abb. 1).

Interessanter als der Befund ist die Genese: Abbildung 1 veranschaulicht nicht nur den Altersaufbau der deutschen Gesellschaft, sondern spiegelt auch die einschneidenden Ereignisse ihrer Geschichte wider: Vor dem Ersten Weltkrieg wies die graphische Darstellung der Altersstruktur noch die für eine vorindustrielle bzw. agrarisch geprägte Bevölkerung typische Form einer „störungsfreien“ Pyramide auf. Die beiden Weltkriege verursachten dann neben der Weltwirtschaftskrise um 1932 Einschnitte und Ausbuchtungen bei den heute etwa 60-, 70- bzw. 85-jährigen.

Peter Kostorz und Friedrich E. Schnapp, Institut für Sozialrecht der Ruhr-Universität Bochum.
Für die hier im Vordergrund stehenden Auswirkungen des demographischen Wandels auf die sozialen Sicherungssysteme ist dann eine Entwicklung interessant, die etwa Mitte der 1960er Jahre einsetzt: Die fast rasante Talfahrt der Geburtenzahlen. Um die „Reproduktion“ der Bevölkerung zu gewährleisten, müsste jede Frau im arithmetischen Mittel knapp 2,2 Kinder zur Welt bringen. ${ }^{8}$ Dieser statistische Wert ist seitdem jedoch kontinuierlich gesunken (Abb. 2); im Jahre 2004 wurden in Deutschland nur noch etwa $61 \%$ derjenigen Kinder geboren, die nötig wären, um den derzeitigen Umfang der Bevölkerung ceteris paribus - also vor allem ohne Migrationen - langfristig zu sichern. ${ }^{9}$

Oftmals wird in diesem Zusammenhang vereinfachend vom „Pillenknick“ gesprochen; der Umstand dieses säkularen Geburtenrückgangs ist jedoch nicht monokausal zu erklären. ${ }^{11}$ In der soziologischen Forschung geht man vielmehr von einem Bedingungsgefüge mit zahlreichen Ursachenkomplexen aus, wobei lange Jahre unklar blieb, welches Gewicht den verschiedenen Faktoren zukommt. Der Bevölkerungswissenschaftler Herwig Birg hat in diesem Zusammenhang die der ökonomischen Lehre entlehnte Theorie der „biographischen Opportunitätskosten" entwickelt: ${ }^{12}$ Danach ist die Gründung einer Familie stets mit dem Verlust persönlicher Flexibilität und Mobilität verbunden, was unweigerlich die Wahlmöglichkeiten der Eltern bezüglich 


\section{Altersaufbau der Bevölkerung in Deutschland}

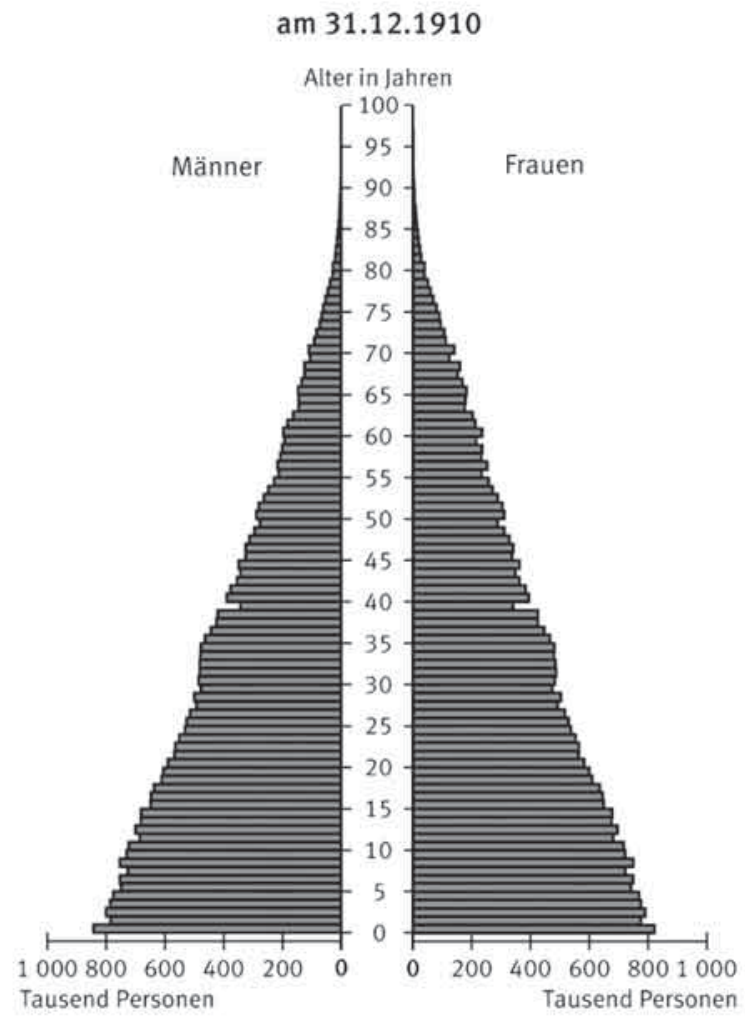

am 31.12.2001

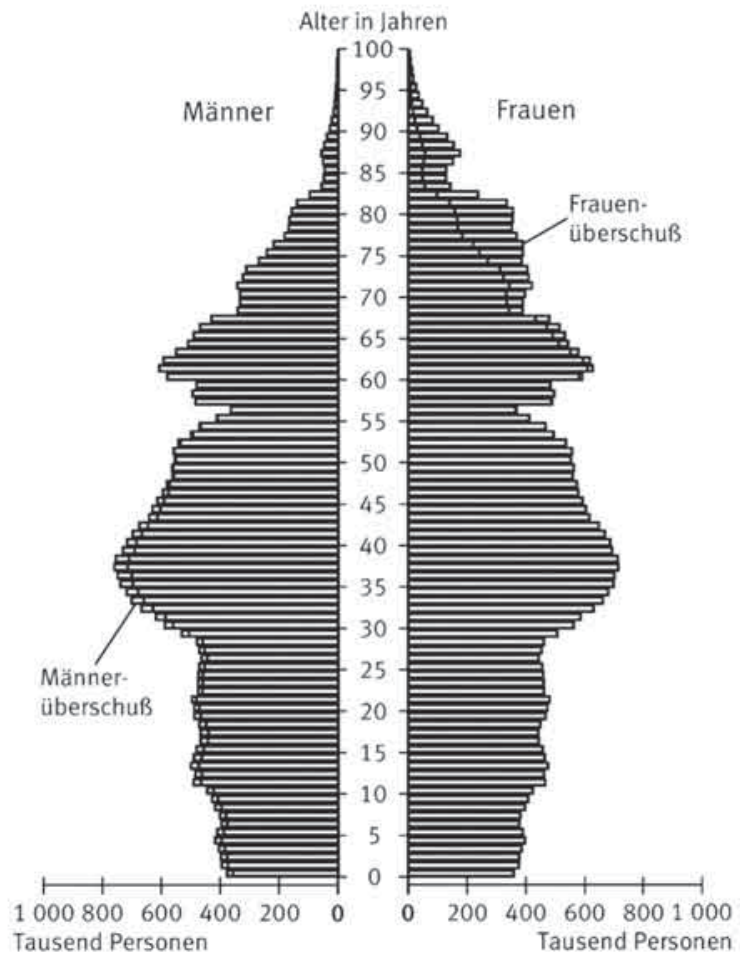

am 31.12.1950

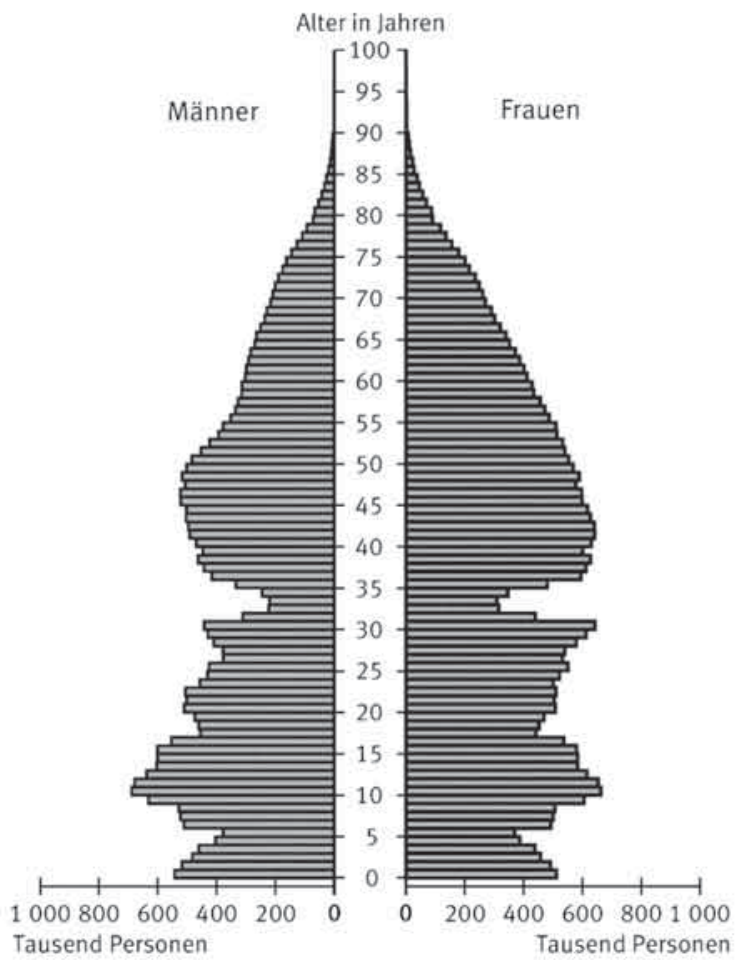

am 31.12.2001 und am 31.12.2050

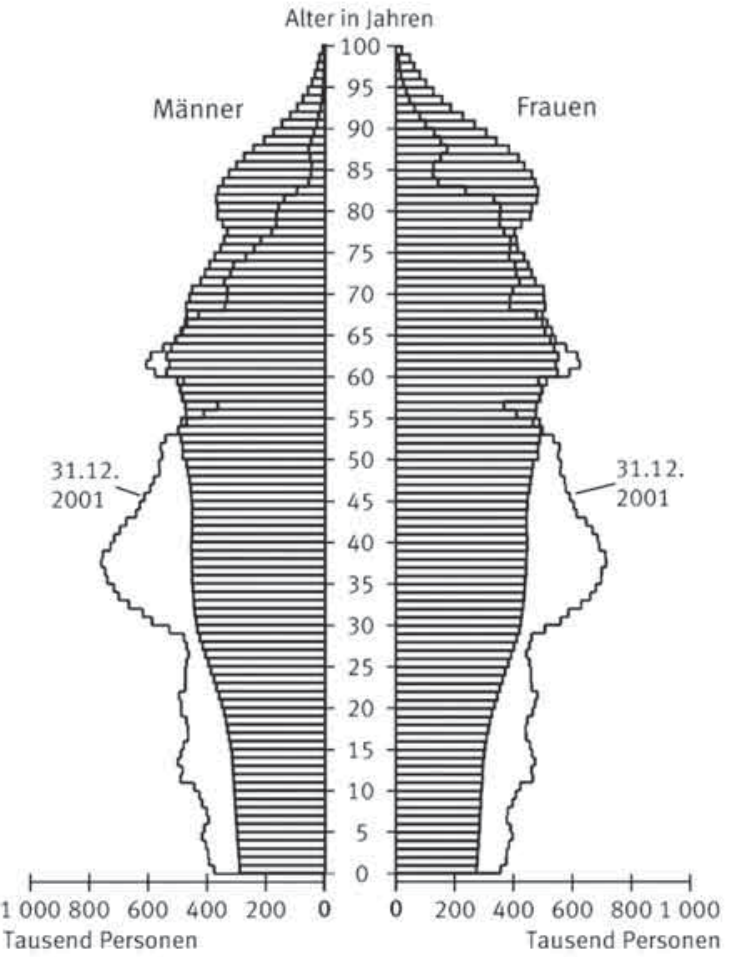


Abbildung 2: Geburtenziffern von 1950 bis 2004

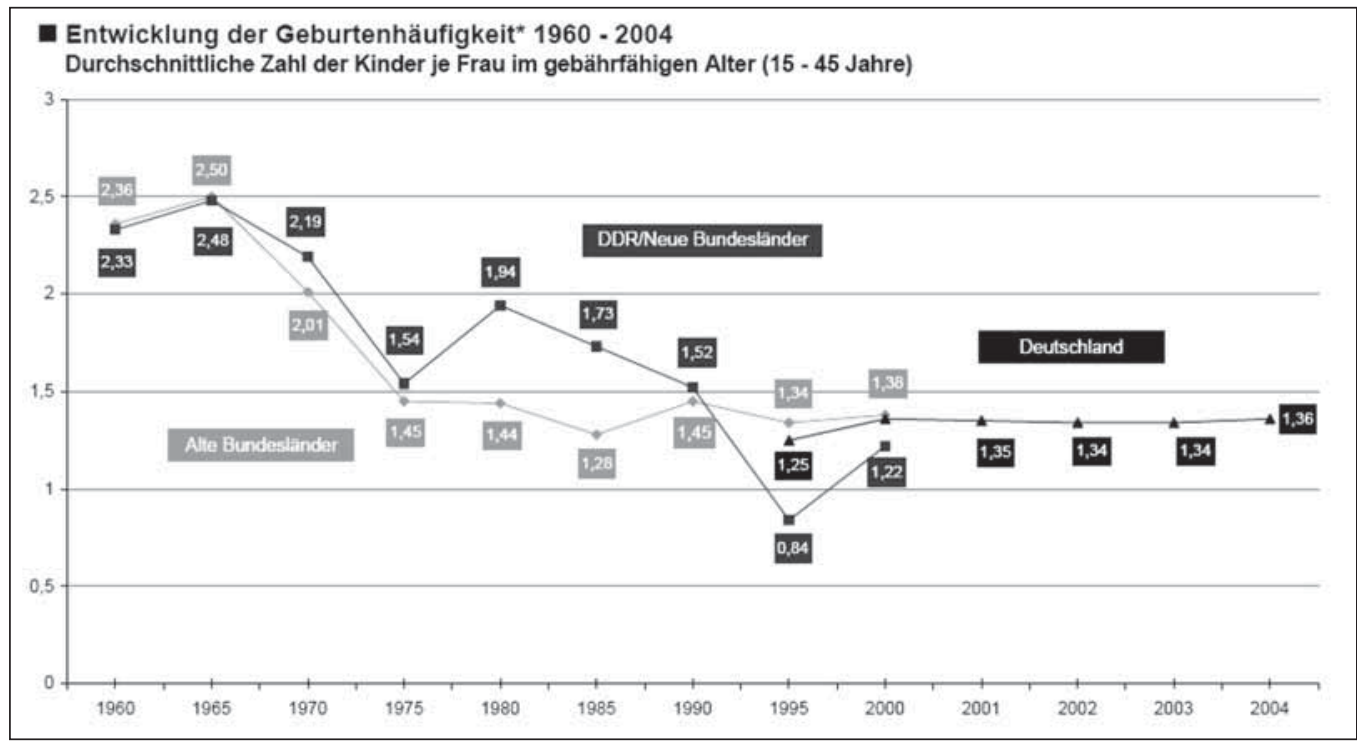

Quelle: www.sozialpolitik-aktuell.de (1. September 2006)

ihrer individuellen Lebensweise und ihre Wettbewerbsfähigkeit auf dem Arbeitsmarkt einschränkt. Da es sich hierbei um eine unumkehrbare Beschneidung persönlicher biographischer Alternativen handelt, wird ein eventuell latent bestehender Kinderwunsch zugunsten persönlicher Unabhängigkeit und der damit verbundenen besseren beruflichen Möglichkeiten immer öfter in den Hintergrund gerückt. ${ }^{13}$

Da eine Änderung dieser gesellschaftlichen Faktoren und der diese determinierenden individuellen biographischen Präferenzen kaum zu erwarten ist, wird die Geburtenrate - hierin sind sich alle Prognosen einig auch in Zukunft auf niedrigem Niveau bleiben oder sogar noch weiter sinken. ${ }^{14}$ Der Umfang der zum Baum mutierten Pyramide wird daher im unteren Teil weiter abnehmen, mit der Folge, dass sich die Wettertanne allmählich in einen Pilz mit breiter Haube und schmalem Stiel auswächst (Abb. 1). ${ }^{15}$

Weiter fällt auf, dass die Tanne bzw. der Pilz zum einen immer weiter in die Höhe wächst und zum anderen in den oberen Segmenten an Breite zunimmt: Immer mehr Menschen erreichen also ein immer höheres Lebensalter. So hat sich in Deutschland die durchschnittliche Lebenserwartung in den letzten 100 Jahren rein statistisch um etwa zwei Drittel erhöht und stieg von 44,8 Jahren zu Beginn des 20. Jahrhunderts auf 74,8 im Jahre 2000 bei

Abbildung 3: Fernere Lebenserwartung im Alter von 60 Jahren

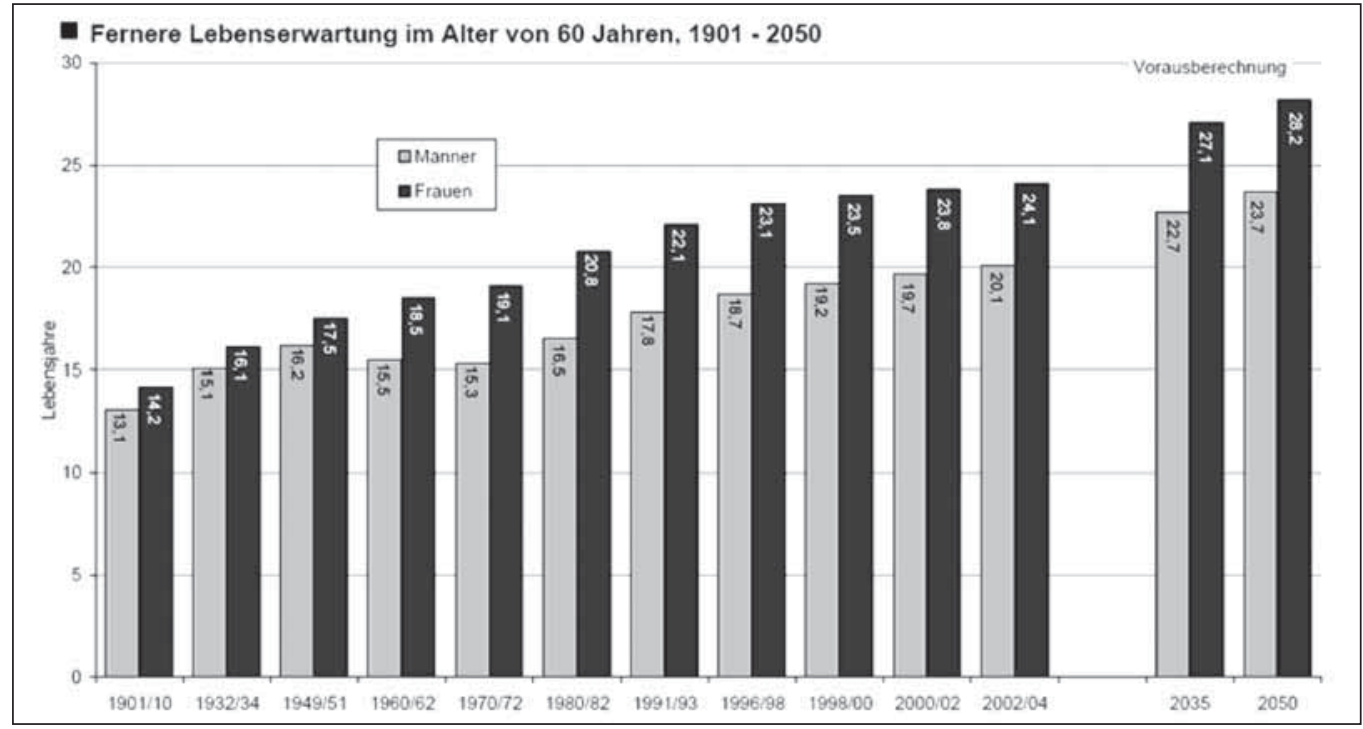

Quelle: www.sozialpolitik-aktuell.de (1. September 2006) 
Abb. 4: Prognostizierte Entwicklung der Altenquotienten

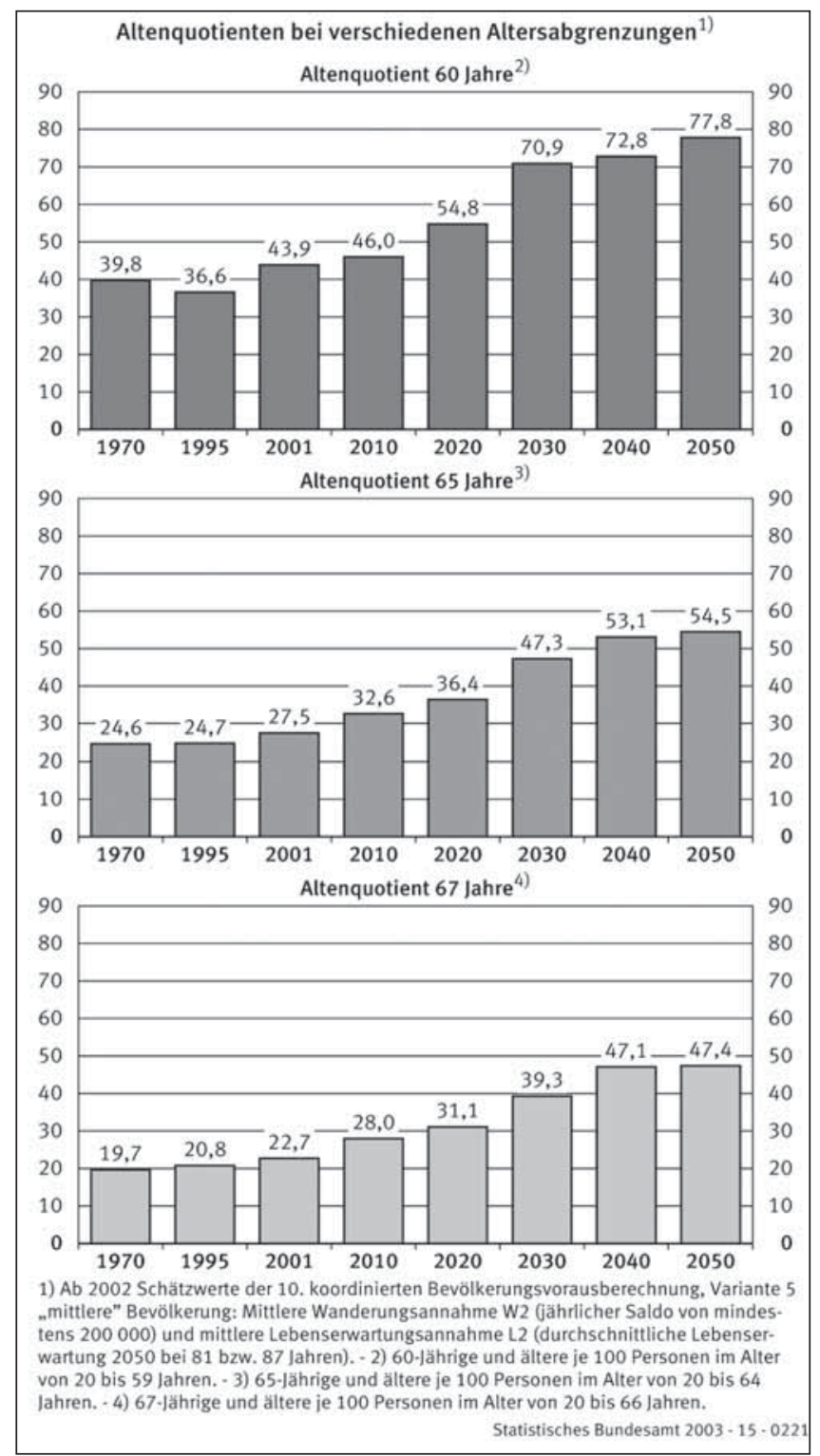

Quelle: Statistisches Bundesamt 2003, 32 einem männlichen Neugeborenen bzw. von 48,3 Jahren auf 80,8 Jahre bei einem neugeborenen Mädchen. ${ }^{16}$ Ebenfalls angestiegen ist die sog. fernere Lebenserwartung, also die Zeit, die ein Mensch ab einem bestimmten Alter noch zu erwarten hat. Dies trifft selbst noch auf sehr alte Menschen zu und ist wesentlich auf den medizinischen Fortschritt und eine allgemeine Verbesserung in den Lebensbedingungen der Menschen zurückzuführen. ${ }^{17}$ Heute hat ein 60 -jähriger Mann statistisch gesehen noch eine weitere, "fernere“ Lebenserwartung von etwa 20 Jahren, eine gleichaltrige Frau von rund 24 Jahren. Gegenüber der Wende vom 19. zum 20. Jahrhundert hat sie sich dabei um etwa sieben Jahre bei den Männern und um etwa zehn Jahre bei den Frauen ausgedehnt. Für die Zukunft wird von einem weiteren Anstieg ausgegangen (vgl. Abb. 3). ${ }^{18}$

Beide Aspekte - Geburtenrückgang auf der einen und steigende (durchschnittliche wie ferne) Lebenserwartung auf der anderen Seite - haben eine sog. demographische Alterung der Bevölkerung zur Folge und zwar in dreierlei Hinsicht: ${ }^{20}$

- Die absolute Anzahl älterer Menschen wird immer mehr zunehmen, während die absolute Anzahl jüngerer sinkt.

- Die sog. fernere Lebenserwartung wird weiter steigen, so dass auch die Zahl der Hochbetagten (oder nach soziologischer Terminologie der sog. „alten Alten“) steigen wird.

- Der relative Anteil Älterer und Hochbetagter an der Gesamtbevölkerung wird zunehmen.

Von besonderer Bedeutung für die weiteren Ausführungen wird der dritte Punkt sein, also die Steigerung des sog. Altenquotienten (Abb. 4). Um den relativen Anteil Älterer an der Gesamtbevölkerung zu verdeutlichen, wird diese in drei Alterskategorien eingeteilt. Es handelt sich dabei um die - in aller Regel noch nicht erwerbstätigen unter 20-jährigen, die Mitbürger im Erwerbsalter und Personen ab dem 60. (zur Berechnung des sog. Altenquotienten $_{60}$ ) bzw. 65. Lebensjahr (zur Berechnung des

Abbildung 5: Aktuelles und prognostiziertes Verhältnis von alten zu jungen Menschen

\begin{tabular}{|c|c|c|c|c|}
\hline Jahr & „Senior & $\begin{array}{c}\text { Personen } \\
\text { zwischen } 20 \text { und } \\
\text { unter } 60 \text { Jahren }^{\text {(Altenquotient }} \text { (60) }\end{array}$ & $\begin{array}{c}\text { Personen } \\
\text { zwischen } 20 \text { und } \\
\text { unter } 65 \text { Jahren }^{\text {(Altenquotient }}{ }_{65} \text { ) }\end{array}$ & $\begin{array}{c}\text { Personen } \\
\text { zwischen } 20 \text { und } \\
\text { unter } 67 \text { Jahren } \\
\text { (Altenquotient }_{67} \text { ) }\end{array}$ \\
\hline 2001 & $\pi 1$ & 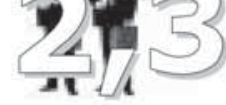 & & \\
\hline 2050 & $\boldsymbol{T}+1$ & & & \\
\hline
\end{tabular}

Quelle: Kostorz 2004, 63 
sog. Altenquotienten 65$) \cdot{ }^{21}$ Seit der Diskussion um eine Anhebung der Regelaltersgrenze von 65 auf 67 Jahren wird darüber hinaus häufig auch der sog. Altenquotient 67 berechnet.Zum Zeitpunkt der letzten Koordinierten Bevölkerungsvorausberechnung des Statistischen Bundesamtes lag der am Sozialrecht orientierte Altenquotient $_{65}$ noch bei 27,5. 100 Menschen im Erwerbsalter standen damit 27,5 Personen im maßgeblichen Alter für eine Regelaltersrente gegenüber. Bis zum Jahr 2050 wird sich dieser Wert auf 54,5 verdoppeln. Die Steigerungsrate des Altenquotienten 60 liegt etwas darunter, diejenige des Altenquotienten $_{67}$ sogar darüber. ${ }^{23}$ Das sind recht abstrakte Zahlen. Anschaulicher wird es, wenn das Verhältnis von Jung zu Alt auf jeweils eine ältere Person heruntergerechnet wird (Abb. 5). Danach entfielen zum genannten Zeitpunkt beispielsweise auf einen "Senior“ im Alter von 65 oder mehr Jahren knapp vier Personen im Alter von 20 bis 65 Jahren; im Jahre 2050 werden es voraussichtlich nicht einmal mehr zwei sein!

\section{Die Auswirkungen auf die gesetzliche Renten- versicherung}

Das System der gesetzlichen Rentenversicherung ist stark von intergenerativen, also sowohl interpersonellen als auch intertemporalen Umlagen und Transfers geprägt: Hier bestreiten die jeweils aktiven Versicherten, die i.d.R. keine Leistungsansprüche haben, mit ihren Beiträgen ${ }^{25}$ die jeweils laufenden Renten der älteren Generation, die als Rentenbezieher grundsätzlich nicht beitragspflichtig sind. Kommen die jetzt erwerbstätigen Kohorten in das maßgebliche Rentenalter, wird ihre Alterssicherung von den dann Beschäftigten finanziert, die damit wiederum Ansprüche gegen die Folgegeneration erwerben und so fort. Dieses sog. Umlageverfahren beruht mithin auf einem „Vertrag“ zwischen der jeweiligen Erwerbstätigengeneration einerseits und der Rentnergeneration andererseits (Abb. 6) ${ }^{26}$

\section{Abbildung 6: Der in der gesetzlichen Renten- versicherung existierende Generationenvertrag}

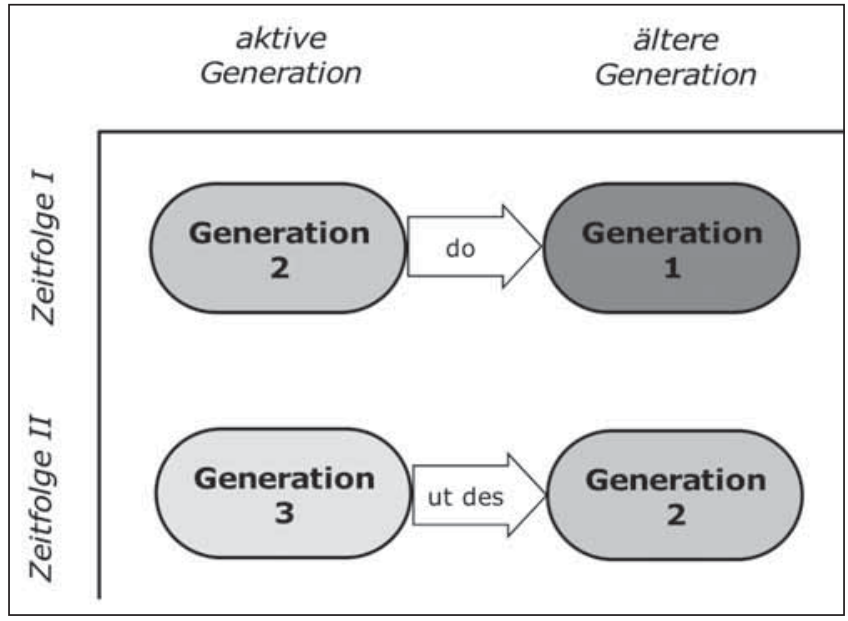

Quelle: Kostorz 2004, 61
Ein solches Umlagesystem ist in erheblichem Maße anfällig für den beschriebenen demographischen Wandel einer Gesellschaft; so wird etwa die massive Verschiebung des Altenquotienten dazu führen, dass in Zukunft einem Rentner immer weniger Beitragszahler gegenüberstehen (vgl. Abb. 5). ${ }^{27}$ Prognosen deuten daher darauf hin, dass der Beitragssatz zur gesetzlichen Rentenversicherung von heute 19,5\% bis zum Jahr 2040 auf über 30\% der Bruttolöhne steigen könnte. ${ }^{28}$

Eine effizientere Finanzierung der Rentenversicherung wird daher oftmals im sog. Kapitaldeckungsverfahren gesehen. ${ }^{30}$ Es charakterisiert ein Alterssicherungssystem, in dem die Mitglieder einer Generation während ihrer Erwerbsphase mittels Ersparnisbildung einen Real- bzw. Finanzkapitalstock aufbauen, der während der Ruhestandsphase im Alter wieder abgebaut und im Idealfall von einem Risikoausgleich abgesehen - bis zum Lebensende verbraucht wird. ${ }^{31}$ Die gebildeten Ersparnisse verzinsen sich dabei mit dem jeweiligen Marktzins; hinzu tritt eine Gewinnbeteiligung, die vom Geschäftserfolg des Versicherungsunternehmens abhängig ist. ${ }^{32}$

Dieses Verfahren wird zum einen deshalb für vorteilhafter gehalten, weil dadurch in der Vergangenheit höhere Renditen für die geleisteten Beiträge erzielt werden konnten als im Umlageverfahren. ${ }^{33}$ Zum anderen soll dieses System die drohenden demographischen Belastungen dadurch besser verkraften können, dass die Versicherten ausschließlich für sich selbst sparen und so von intergenerativen Umverteilungseffekten unabhängig sind. Genau das könnte sich jedoch als fromme Wunschvorstellung erweisen: Schon im Jahre 1952 wies der Nationalökonom Gerhard Mackenroth darauf hin, „daß aller Sozialaufwand immer aus dem Volkseinkommen der laufenden Periode gedeckt werden muß. Es gibt keine andere Quelle und hat nie eine andere Quelle gegeben, aus der Sozialaufwand fließen könnte, es gibt keine Ansammlung von Fonds, keine Übertragung von Einkommensteilen von Periode zu Periode, kein »Sparen« im privatwirtschaftlichen Sinne - es gibt einfach gar nichts anderes als das laufende Volkseinkommen als Quelle für den Sozialaufwand. “34 Nach dieser These wäre also auch das Kapitaldeckungsverfahren nicht vor den Auswirkungen des beschriebenen demographischen Wandels gefeit: So muss das akkumulierte Kapital zur Renditeerzielung investiert und in der Entsparphase wieder veräußert werden. Bei einer Bevölkerungsabnahme geht die Nachfrage nach Kapital jedoch in etwa dem gleichen Maße zurück, so dass sich der Preis für das Kapital und damit auch die Rendite der Rücklagen ebenso wie die erwarteten Veräußerungsgewinne stark mindern könnten. ${ }^{35}$

Die Frage, ob ein Kapitaldeckungsverfahren mikround/oder makroökonomisch effizienter ist als das bestehende Umlageverfahren kann jedoch insofern dahinstehen, als neben den ökonomischen Ungewissheiten gewichtige verfassungsrechtliche Bedenken gegen eine vollständige Umstellung des Umlage- auf ein Kapitaldeckungsverfahren sprechen: Seit einer Entscheidung des Bundesverfassungsgerichts aus dem Jahre $1980^{36}$ ist es all- 
Abbildung 7: Belastungsquoten im Umlageverfahren und im Kapitaldeckungsverfahren

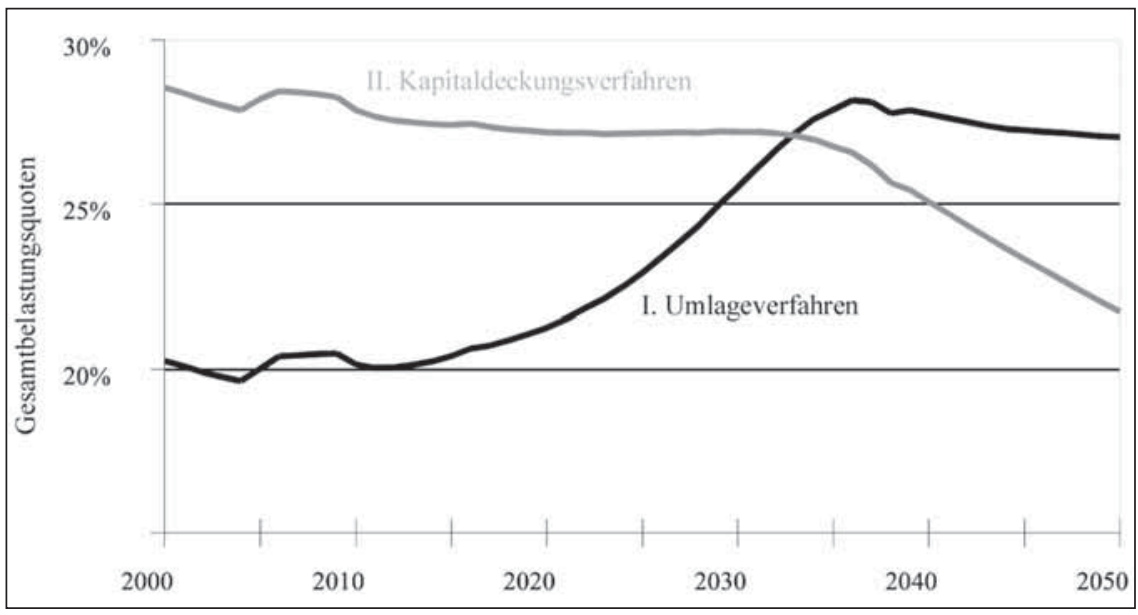

Quelle: Kostorz 2004, 64

gemein anerkannt, dass renten- bzw. sozialversicherungsrechtliche Ansprüche dem Eigentumsschutz des Art. 14 GG unterliegen, wenn es sich hierbei um eine „vermögenswerte Rechtsposition“ handelt, „die nach Art eines Ausschließlichkeitsrechts dem Rechtsträger als privatnützig zugeordnet ist, ... auf nicht unerheblichen Eigenleistungen des Versicherten beruht und zudem der Sicherung seiner Existenz dient ${ }^{\text {“37 }}$. Dies trifft nach allgemeiner Auffassung in jedem Fall auf die im Laufe des Erwerbslebens eines Versicherten „angesammelten“ oder „erarbeiteten“ Entgeltpunkte $\mathrm{zu}^{38}{ }^{38}$ die im Alter einen Anspruch auf Rente zwar nicht in Höhe einer bestimmten Relation zu den geleisteten Beiträgen, so aber im Verhältnis des individuellen Verdienstes (bis zur Beitragsbemessungsgrenze) zum jeweiligen Durchschnittsverdienst aller aktiven Versicherten begründen. ${ }^{39}$ Art. 14 GG lässt damit zwar die Einführung eines reinen Kapitaldeckungsverfahrens für Generationen zu, die noch nicht dem derzeitigen System der gesetzlichen Rentenversicherung angehören, nicht jedoch eine abrupte Umstellung für aktive Versicherte oder gar Rentner. ${ }^{40}$

Eine Umstellung vom Umlage- auf das Kapitaldeckungsverfahren könnte folglich nur in Form eines gleitenden Übergangs bewerkstelligt werden, wobei die von Art. 14 GG geschützten Rechtspositionen zu berücksichtigen wären. Probleme ergäben sich jedoch stets bei der Finanzierung der laufenden, umlagefinanzierten Renten, da hierfür ab dem Zeitpunkt des Eintritts in das Kapitaldeckungsverfahren keine Beiträge mehr zu entrichten wären - diese Gelder würden bei einer Systemumstellung für den Aufbau des Kapitalstocks benötigt. Die Auszahlung der Bestandsrenten müsste daher durch Staatszuschüsse an die Rentenversicherung sichergestellt werden, was wiederum die Erhebung von Steuern bei denjenigen Generationen erforderlich machte, deren Alterssicherung über das Kapitaldeckungsverfahren erfolgt. Für eine Zeit von etwa 30 bis 40 Jahren käme es m.a.W. zu einer Doppelbelastung der jeweils erwerbstätigen Generation: Neben dem beitragsfinanzierten Aufbau des Kapitalstocks müssten über Steuern die noch bestehenden Rentenansprüche der vorherigen Erwerbsgeneration bedient werden. ${ }^{41}$

Abbildung 7 simuliert die unterschiedlichen Belastungsverläufe: ${ }^{42}$ Kurve I. illustriert den Verlauf bei einem Verbleib im Umlageverfahren. Der Beitragssatz zur gesetzlichen Rentenversicherung stiege danach kontinuierlich auf etwa $30 \%$ an. Der mit II. gekennzeichnete Belastungspfad simuliert einen vollständigen Übergang zum Kapitaldeckungsverfahren ab dem Jahr 2000, kombiniert mit einer (steuerlichen) Finanzierung der verfassungsrechtlich geschützten "Altlasten" aus dem abgeschafften Umlageverfahren. Die Kurve weist die jeweilige Gesamtbelastung in Prozent des Erwerbseinkommens aus, bestehend aus den notwendigen Sparleistungen für den Aufbau des Kapitalstocks einerseits und dem erhöhten Steueraufkommen für die Erfüllung der durch Art. 14 GG geschützten Rechtspositionen aus dem Umlageverfahren andererseits: „It can be seen that the transfer to full capital funding takes the system out of the frying pan into the fire, because it is a mirror image of pay-as-you-go path. ${ }^{43}$ Die im Umlagesystem ab etwa 2035 auftretenden Belastungen von mehr als $28 \%$ würden in die Gegenwart verlagert; umgekehrt würde die heutige Beitragsbelastung bei einem Übergang zum Kapitaldeckungsverfahren etwa im Jahr 2055 erreicht und dann langfristig auf rund $18 \%$ sinken. ${ }^{44}$

In beiden Fällen würde also ausschließlich die erwerbstätige Generation belastet - die jeweilige Rentnergeneration hätte keine oder nur geringe Einbußen hinzunehmen. Um beurteilen zu können, ob dies im Sinne einer ausgleichenden Generationengerechtigkeit sinnvoll wäre, ist an die Ausführungen zur Ausgestaltung des der gesetzlichen Rentenversicherung zugrunde liegenden Generationenvertrages anzuknüpfen (Abb. 6), der nach geltender Rechtslage als Zweigenerationenvertrag zwischen der jeweiligen aktiven Altersgruppe einerseits und der betreffenden Rentnergeneration andererseits 
Abbildung 8: Dreigenerationenvertrag nach Oswald von Nell-Breuning

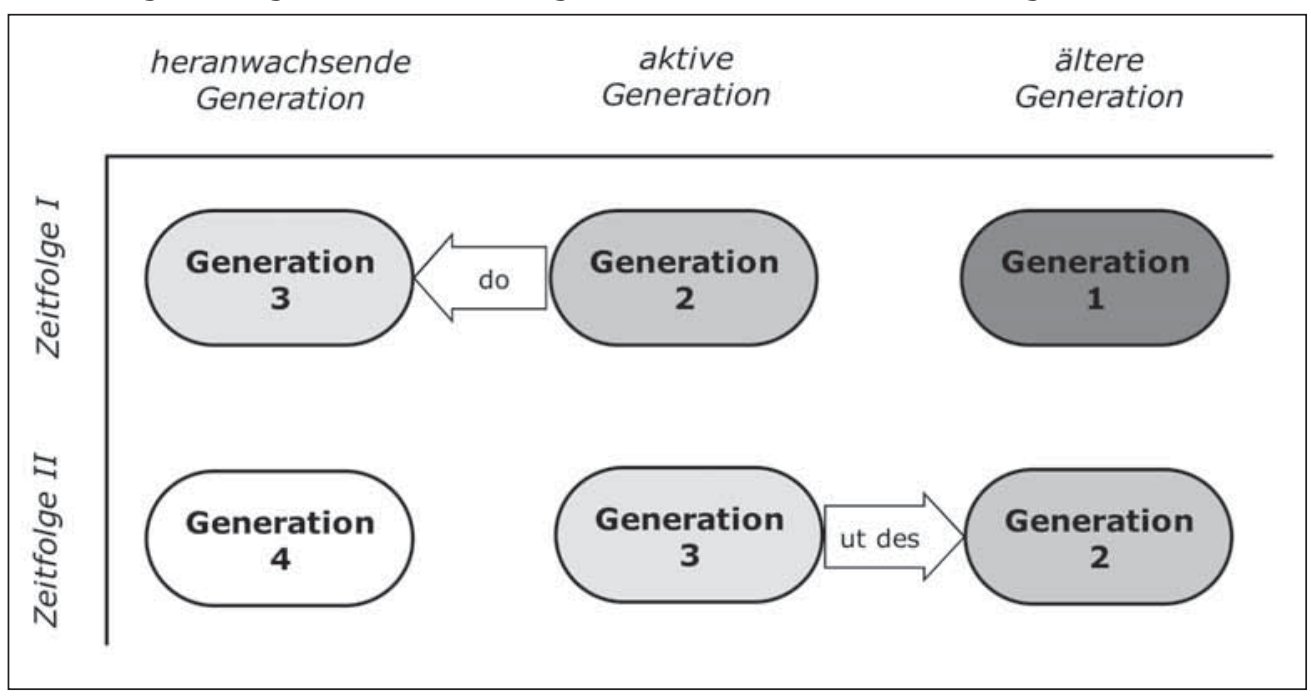

Quelle: Kostorz 2004, 65

charakterisiert wurde. ${ }^{46}$ Oswald von Nell-Breuning, der das deutsche Rentenversicherungssystem notabene stets als ein System zur Prämierung von Kinderlosigkeit angeprangert hat, skizzierte die „Rechtsbeziehungen“ zwischen den Generationen bereits im Vorfeld der Rentenreform 1957, die den Umstieg vom ursprünglichen Kapitaldeckungs- zum heutigen Umlageverfahren vollzog, gesellschafts- und sozialpolitisch richtigerweise als synallagmatische Beziehung zwischen allen Generationen und plädierte entsprechend für einen Dreigenerationenvertrag. ${ }^{47}$ Dieses Modell eines alle Generationen berücksichtigenden Gesellschaftsvertrages geht davon aus, dass der Vertragsinhalt nicht nur die Versorgung der älteren durch die aktive Generation umfasst, sondern ebenso die Erziehung der heranwachsenden (Abb. 8). ${ }^{48}$

Derzeit sind Alleinstehende und kinderlose Paare zwar nicht oder in nicht nennenswertem Umfang an der Versorgung und Erziehung der nachfolgenden Generation beteiligt, erwerben nach dem Modell des Zweigenerationenvertrages aber dennoch volle Rentenansprüche gegen sie. ${ }^{50}$ Anders wäre die „Rechtslage“ bei einer Zugrundelegung des Dreigenerationenvertrages: Hiernach sind die jeweils älteren Generationen insofern „vertragsbrüchig“ geworden, als sie aufgrund ihres geänderten generativen Verhaltens die „Reproduktion“ der Bevölkerung vernachlässigt haben, so dass sie auch nicht auf eine uneingeschränkte Unterstützung der dann jeweils aktiven Generation bauen dürften. ${ }^{51}$ Die durch die demographische Entwicklung bedingte unausweichliche Mehrbelastung darf also nicht allein den jeweils aktiven Versicherten aufgebürdet werden, sondern muss durch einschneidendere Änderungen des Rentenrechts als bisher zwischen ihnen und der Rentnergeneration aufgeteilt werden. ${ }^{52}$

Unter diesem Gesichtspunkt erscheint sowohl eine Kürzung der Rentenbezugsdauer durch eine Verlängerung der Lebensarbeitszeit als auch eine Reduzierung des durchschnittlichen Rentenzahlbetrages mittels einer Ab- senkung des allgemeinen Rentenniveaus unausweichlich. Nur so kann den beiden großen Ursachenkomplexen der demographischen Alterung unserer Gesellschaft - der steigenden Lebenserwartung einerseits und der Erosion der Geburtenrate andererseits - effektiv und generationengerecht begegnet werden. ${ }^{53}$

Eine Reduktion der Rentenbezugsdauer wurde bereits durch die letzte CDU/CSU-FDP-Bundesregierung eingeleitet, die damit begann, das Renteneintrittsalter für bestimmte Rentenarten teilweise nach hinten zu verlagern, die Lebensarbeitszeit also zu verlängern; ${ }^{54}$ im Falle eines vorzeitigen Rentenbezugs sieht das Rentenrecht seitdem Abschläge vor. ${ }^{55}$ Ähnliches bezweckte die ihr nachfolgende rot/grüne Bundesregierung, die die gesetzlichen Anspruchsvoraussetzungen für den Bezug einer Erwerbsminderungsrente ganz erheblich verschärfte und für deren Gewährung eine Befristungsregelung einführte. ${ }^{56}$ Durch diese Maßnahmen soll sich das effektive Renteneintrittsalter von derzeit etwa 60 Jahren erhöhen und sich der Altersgrenze für die gesetzliche Regelaltersrente von 65 Jahren immer weiter annähern. Im Jahre 2050 werden einem Rentner dadurch voraussichtlich nicht nur 1,3, sondern immerhin 1,8 Menschen im erwerbsfähigen Alter gegenüberstehen (vgl. Abb. 5).

Was bislang ausblieb, ist indes eine generelle Anhebung der besagten Regelaltersgrenze von derzeit 65 Jahren. Doch auch dies wird unausweichlich sein: Allein in den letzten 20 Jahren hat sich die durchschnittliche fernere Lebenserwartung 65-jähriger um über zwei Jahre erhöht; ${ }^{57}$ für die Zukunft zeichnet sich ein weiterer Anstieg ab. ${ }^{58}$ Mit Recht lässt sich daher die Frage stellen, warum die gewonnene Lebenszeit (wie bisher) ausschließlich zu einer Ausdehnung der Rentenphase führen und sie nicht zwischen Renten- und Erwerbsphase aufgeteilt werden sollte. ${ }^{59}$ Hierfür spricht auch, dass Rentner zum Zeitpunkt des Renteneintritts im Vergleich zu früheren Zeiten wesentlich rüstiger und aktiver sind, so dass eine 
angemessen längere Berufstätigkeit nicht zwangsläufig mit einer Einschränkung der Lebensqualität zu Beginn der Ruhestandsphase verbunden sein muss. ${ }^{60}$ Sowohl die Rürup-, als auch die Herzog-Kommission schlugen daher vor, die Regelaltersgrenze langfristig auf 67 Jahre heraufzusetzen. ${ }^{61}$ Dadurch könnte das Verhältnis von potentiellen Leistungsempfängern und Beitragszahlern im Jahre 2050 zwar nicht auf dem heutigen Niveau von 1 zu 2,3 stabilisiert werden, doch läge es mit $1 \mathrm{zu} 2,1$ auch nicht mehr beängstigend darunter (vgl. Abb. 5). ${ }^{62}$ Eine endgültige Entscheidung darüber hat die Politik bislang jedoch noch nicht getroffen.

Doch auch diese Maßnahmen - das zeigt der Vergleich des heutigen Altenquotienten 60 mit dem Altenquotienten $_{65}$ des Jahres 2050 - können die Folgen der Alterung der Gesellschaft nicht ganz ausgleichen. Zusätzlich erforderlich ist vielmehr eine Absenkung des Rentenniveaus, welches - vereinfacht gesagt - angibt, wie hoch die ausgezahlten Renten im Vergleich zum Erwerbseinkommen der rentenversicherungspflichtigen Beschäftigten sind, die die laufenden Renten im Umlageverfahren mit ihren Beitragszahlungen aufzubringen haben. Da Art. 14 GG bestehende Rentenanwartschaften - wie dargestellt - nicht in ihrer absoluten Höhe gegen gesetzgeberische Eingriffe schützt, sind entsprechende Maßnahmen aus verfassungsrechtlicher Sicht sowohl für die aktiven Versicherten als auch für die Bestandsrentner grundsätzlich gerechtfertigt. ${ }^{63}$ Eine solche Minderung des Rentenniveaus bewirkte bereits die Novellierung der Rentenanpassungsformel durch die Rentenreform 2001, die die Dynamisierung der Renten auf die sog. modifizierte Bruttoanpassung umstellte. ${ }^{64}$ Zudem wird seit dem Jahr 2005 ein auf die Rürup-Kommission zurückgehender sog. Nachhaltigkeitsfaktor bei der Rentenanpassung einkalkuliert. ${ }^{65}$ Er berücksichtigt das Verhältnis zwischen Beitragszahlern und Rentenempfängern und bewirkt, dass der steigende Anteil von Rentnern die jährliche Rentenanpassung dämpft. Dadurch

Abbildung 9: Schematische Darstellung individueller Alters-Ausgabenprofile nach der Medikalisierungstheorie und der Kompressionstheorie

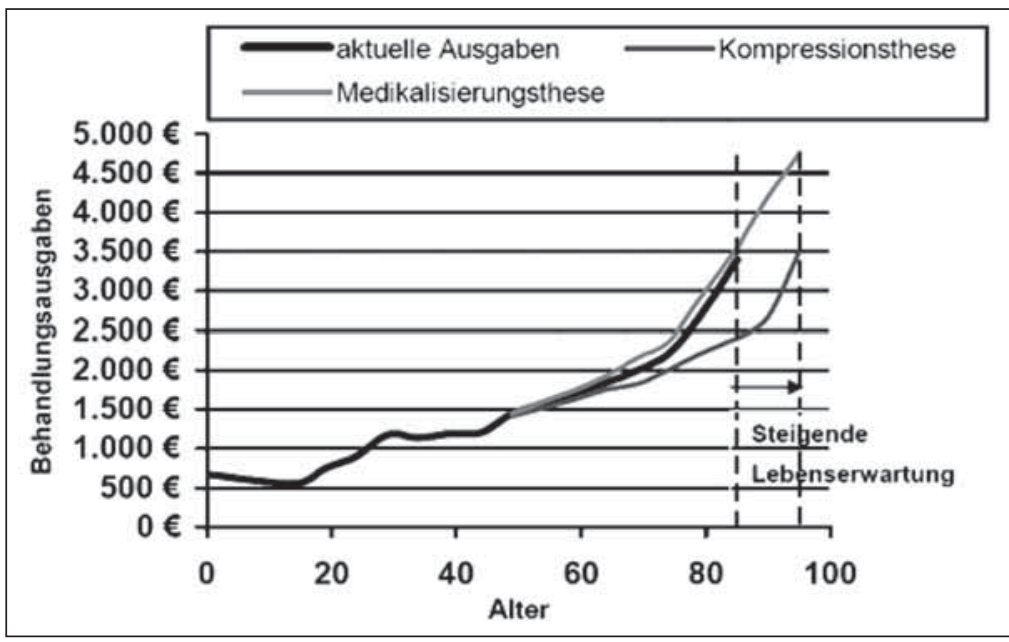

Quelle: www.hausarbeiten.de (1. September 2006) kommt es zu einer beachtlichen Verlangsamung des Rentenanstiegs im Vergleich zur Lohnentwicklung. Die Renten werden also auch weiterhin steigen, allerdings nicht mehr im Gleichschritt mit den Löhnen und Gehältern, sondern abgeschwächt entsprechend der fortschreitenden Alterung der Gesellschaft. ${ }^{66}$

Zum Vergleich unterschiedlicher Rentenniveaus bedient sich die Versicherungsmathematik in der gesetzlichen Rentenversicherung eines sog. Standard- oder Eckrentners, der über 45 Versicherungsjahre hinweg ein Arbeitsentgelt erzielt hat, das jeweils exakt dem Durchschnittsverdienst eines rentenversicherungspflichtigen Beschäftigten entsprochen hat. Dessen Bruttorentenniveau betrug zum Zeitpunkt der letzten Erhebung im Jahre 2004 48,6\%. ${ }^{67}$ Bis zum Jahr 2040 soll dieses Rentenniveau durch die Veränderungen in der Rentenanpassungsformel auf 38,4\%, unter Berücksichtigung einer Verlängerung der Lebensarbeitszeit um zwei Jahre auf 40,1\% sinken. ${ }^{68}$

Diese sog. Rentenlücke muss durch private Vorsorge ausgeglichen werden. ${ }^{69} \mathrm{Um}$ hierzu entsprechende Anreize zu schaffen, haben Versicherte die Möglichkeit, mit dem Abschluss eines sog. Riester-Sparplans in den Genuss von Steuererleichterungen und einer staatlichen Förderung in Form einer Grund- und einer Kinderzulage zu kommen. ${ }^{70}$ Modellrechnungen gehen davon aus, dass ein Standardrentner, der immer den vollen Eigenbeitrag geleistet und damit auch die volle staatliche Förderung erhalten hat, im Jahr 2040 eine Riester-Rente mit einem Bruttorentenniveau von $9 \%$ erhalten wird. Zusammen mit der gesetzlichen Rente käme er dann auf ein Gesamtversorgungsniveau von $49 \%$ brutto, was in etwa dem heutigen Niveau einer alleinigen gesetzlichen Rente entspricht. ${ }^{71}$

\section{Die Auswirkungen auf die gesetzliche Krankenversicherung}

Bevor darauf eingegangen wird, was der empirische Befund der Bevölkerungsentwicklung in Deutschland für die gesetzliche Krankenversicherung bedeutet, seien einige Anmerkungen epidemiologischer Art vorausgeschickt, also zur Frage altersbedingter Veränderungen von Krankheitsbildern, und zu daraus resultierenden Auswirkungen auf die Kostenentwicklung im Gesundheitswesen.

Man unterscheidet generell zwischen dem subjektiven und dem objektiven Gesundheitszustand sich in Behandlung begebender $\mathrm{Pa}-$ tienten bzw. Versicherter. Mit fortschreitendem Lebensalter verschlechtert sich zunächst „nur“ der subjektive Gesundheitszustand, also die gesundheitliche Selbsteinschätzung der Betroffenen. ${ }^{72}$ Für die hier behandelte Fragestellung ist dies deshalb zentral, weil subjektives Unwohlsein einen Zugangspfad zum System der medizinischen Versorgung darstellt, was zwangsläufig wiederum zu einer Kosten- 
belastung der gesetzlichen Krankenversicherung führt. ${ }^{73}$ Als weiterer altersspezifischer „Risikofaktor" kommt hinzu, dass speziell bei älteren Patienten mit einer Zunahme der Polypragmasie, also des sog. doctor-hoppings zu rechnen ist, ${ }^{74}$ was nicht nur zu kaum noch kontrollierbaren Nebeneffekten für ihre gesundheitliche Entwicklung insgesamt, ${ }^{75}$ sondern auch zu einer signifikanten Mengenausweitung bei den Leistungen führt. ${ }^{76}$

Was den objektiven Gesundheitszustand betrifft, werden in der sozialmedizinischen Forschung zwei konträre Theorien diskutiert, nämlich die sog. Medikalisierungsthese $^{77}$ einerseits und die Kompressionsthese ${ }^{78}$ andererseits (Abb. 9).

Nach der Medikalisierungsthese ist mit steigendem Alter auch ein verstärkter physischer Alterungs- und damit -abbauprozess verbunden: „Danach ist das, was bei Zunahme der ferneren Lebenserwartung hinzugewonnen wird an Lebenszeit, etwa zu zwei Drittel "gute« Zeit, zu etwa einem Drittel aber eher eine Art »schlechte « Zeit, zumindest vor dem Hintergrund des zu erwartenden Krankheitsbildes und dem damit verknüpften Ausmaß an körperlicher Autonomie und seelischer Funktionstüchtigkeit. Je größer die Lebenserwartung, umso kränklicher die dann lebende ältere Bevölkerung, kränklicher, weil sie eben länger lebt. " ${ }^{80}$ Die These wird durch Untersuchungen gestützt, nach denen im Alter zum einen die Multimorbidität der Patienten steigt und sich zum anderen Krankheiten öfter chronifizieren. ${ }^{81}$ Aus der verstärkten Inanspruchnahme medizinischer Leistungen einerseits sowie dem verlängerten Leistungsbezug aufgrund der ausgedehnten Restlebenszeit andererseits resultiere - so die These - eine überproportionale Beanspruchung von Leistungen im höheren Alter. ${ }^{82}$
Die Kompressionstheorie kommt zu einem anderen Ergebnis. Danach könne die Auftrittswahrscheinlichkeit ebenso wie die Dauer von Krankheiten bei alten Menschen durch den stetigen medizinischen Fortschritts künftig „immer mehr verringert oder zumindest so verlangsamt werden, daß sich die virulente Manifestation der Erkrankungen auf einen immer kürzeren Zeitraum verschieben ließe (»compression of morbidity«)"83. Diese „compressed morbidity“ würde erst unmittelbar vor dem Lebensende ausbrechen, so dass eine weitere Steigerung der (fernen) Lebenserwartung nicht mit einer signifikanten Veränderung des Ausgabenprofils ältere Patienten bzw. Versicherter in den Jahren vor ihrem Tod einherginge. ${ }^{84}$

Um eine dieser beiden Auffassungen bestätigen zu können, ist die derzeitige Datenlage noch zu unsicher, insbesondere wegen fehlender Längsschnittdaten. ${ }^{85}$ Welche These sich verifizieren lassen wird, kann in vorliegendem Zusammenhang jedoch insofern dahinstehen, als beide Theorien nur auf einen Teilaspekt des dreifachen Alterns der Gesellschaft - nämlich auf die stetig wachsende (ferne) Lebenserwartung - und auf daraus resultierende Veränderungen individueller Alters-Ausgabenprofile abheben. Da es aber als gesichert gelten kann, dass ältere Menschen im Durchschnitt generell höhere Ausgaben für Gesundheitsleistungen verursachen als jüngere (Abb. 10), ${ }^{86}$ wird die demographische Entwicklung, die von einer Verschlechterung der Relation von jungen, tendenziell gesunden und damit kostengünstigen zu alten, eher kranken und teuren Versicherten gekennzeichnet ist, ungeachtet künftiger Veränderungen individueller Ausgabenprofile in jedem Fall zu einer signifikanten Steigerung des Beitragssatzes führen (müssen).

Abbildung 10: Behandlungskosten nach Alter und Geschlecht (Krankheitskosten nach Geschlecht und Alter, Durchschnittliche Kosten pro Person in Euro, Deutschland 2002)

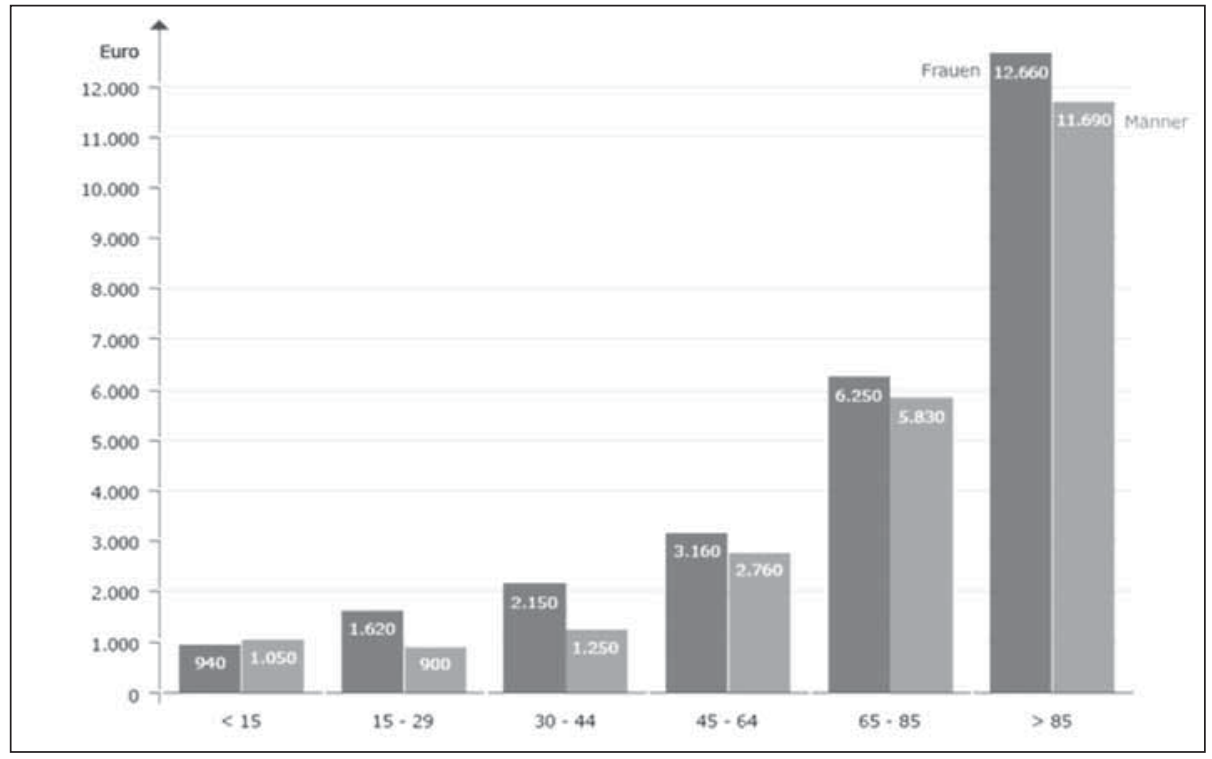

Quelle: www.bpb.de (1. September 2006) 
Schon jetzt verursachen Rentner - obwohl sie nur $26 \%$ der Versicherten stellen - 49,9\% aller Leistungsausgaben (Abb. 11). Die annähernde Verdoppelung des Altenquotienten $_{60}$ bis zum Jahre 2050 bedeutet demzufolge, dass sich in der Mitte des 21. Jahrhunderts jeder Rentner ceteris paribus einen „privaten Finanzierer" unter den dann noch arbeitenden Menschen „suchen“ muss, während heute noch drei bis vier beschäftigte Versicherte ein Kassenmitglied in der Krankenversicherung der Rentner (KVdR) finanzieren. ${ }^{88}$ Allein demographisch bedingt d.h. ohne Berücksichtigung weiterer Faktoren, wie beispielsweise Fortschritte in der Medizin oder der technischen Entwicklung - könnte der durchschnittliche Beitragssatz der gesetzlichen Krankenversicherung in den nächsten 30 bis 40 Jahren von derzeit 14,2\% auf deutlich über $16 \%$ steigen. ${ }^{89}$

Auch in der gesetzlichen Krankenversicherung stellt sich daher unter systematischen Gesichtspunkten die Frage der Generationengerechtigkeit des klassischen Umlageverfahrens. Empirisch betrachtet werden die solidarischen Transfers zu den Rentnern als NettoEmpfängern des Systems ${ }^{91}$ im Allgemeinen begrüßt. ${ }^{92}$ Die „Solidaritätswürdigkeit“ älterer Versicherter wird von den (jüngeren) Nettozahlern - abgesehen von einem offensichtlich generell vorherrschenden Common sense, objektiv Kränkere mitzufinanzieren - vor allem mit einer hohen Erwartungsreziprozität begründet. ${ }^{93}$ „[D]iesem Typus von Reziprozitätsvorstellungen [liegen] im Kern Erwartungen intertemporaler bzw. quasi-intertemporaler (intergenerationeller) Umverteilungen zugrunde ${ }^{\prime 94}$. Wie in der gesetzlichen Rentenversicherung basiert die Solidarität zwischen Jung und Alt mithin auf der Idee des Zweigenerationenvertrages: Die jeweils Beschäftigten stehen für die höheren und in Bezug auf die geleisteten Beiträge nicht äquivalenten Gesundheitsausgaben älterer Versicherter ein, in der Erwartung, die ihnen nachfolgende „virtuelle“ Generation werde eine entsprechende Solidarität üben. ${ }^{95}$ Ebenfalls analog zum Rentensystem wird diesen Generationen jedoch kein vergleichbares, sondern ein erheblich höheres Opfer abverlangt: Auch das
Umlageverfahren in der gesetzlichen Krankenversicherung basiert auf der Prämisse einer „gesunden“ Altersstruktur der Bevölkerung. Da diese Voraussetzung nicht gegeben ist, müssen die jeweils berufstätigen Versicherten immer höhere Beitragslasten aufbringen, um ceteris paribus ein gleich bleibendes Versorgungsniveau der älteren Generation sicherzustellen. Zu vertreten hat dies - nach dem dargestellten Dreigenerationenvertrag nach Nell-Breuning - jedoch nicht die stärker belastete aktive, sondern die jeweils ältere Generation. ${ }^{96}$ Konsolidierungsmaßnahmen im Bereich der gesetzlichen Krankenversicherung müssen unter diesem Gesichtspunkt - wenn auch nicht ausschließlich, so doch aber tendenziell stärker zu Lasten älterer Versicherter gehen.

Konstituierend für die Rechtsbeziehungen zwischen den gesetzlichen Krankenkassen und ihren Versicherten ist zunächst die Mitgliedschaft (oder eine Familienversicherung nach § 10 SGB V), die ihren Rechtsgrund in der Zugehörigkeit der betroffenen Person zum versicherungspflichtigen bzw. -berechtigten Personenkreis nach $\$ \$ 5 \mathrm{ff}$. SGB V hat. Darauf gründen sich einerseits die Leistungsansprüche der Versicherten nach $\S \S 11 \mathrm{ff}$. SGB V, andererseits ihre Beitrags(tragungs)pflichten nach den $\S \S 249$ ff. SGB V. Reformvorschläge zur stärkeren Beteiligung der älteren Generation an den negativen Folgen des demographischen Wandels liegen bislang nahezu ausschließlich für den Bereich des Leistungs- und weniger für den des Beitragsrechts vor.

Bezogen auf den Leistungskomplex wird vor allem vorgeschlagen, die Selbstbeteiligungen zu gesundheitsbezogenen Sozialleistungen zu erhöhen. Diese Vorschläge liegen insofern nahe, als das Ausgabenprofil älterer Versicherter in erster Linie durch eine vermehrte Inanspruchnahme von Krankenhausbehandlung und Arzneimitteln geprägt ist. ${ }^{97}$ Eine verstärkte Eigenbeteiligung in diesen Versorgungsbereichen ${ }^{98}$ würde also tendenziell zu Lasten älterer Kohorten gehen. Problematisch ist jedoch, dass diesem Lösungsansatz insofern äußerst enge Grenzen gesetzt sind, als Rentnerinnen und Rentner über-

Abbildung 11: Generationensolidarität in der gesetzlichen Krankenversicherung 2005

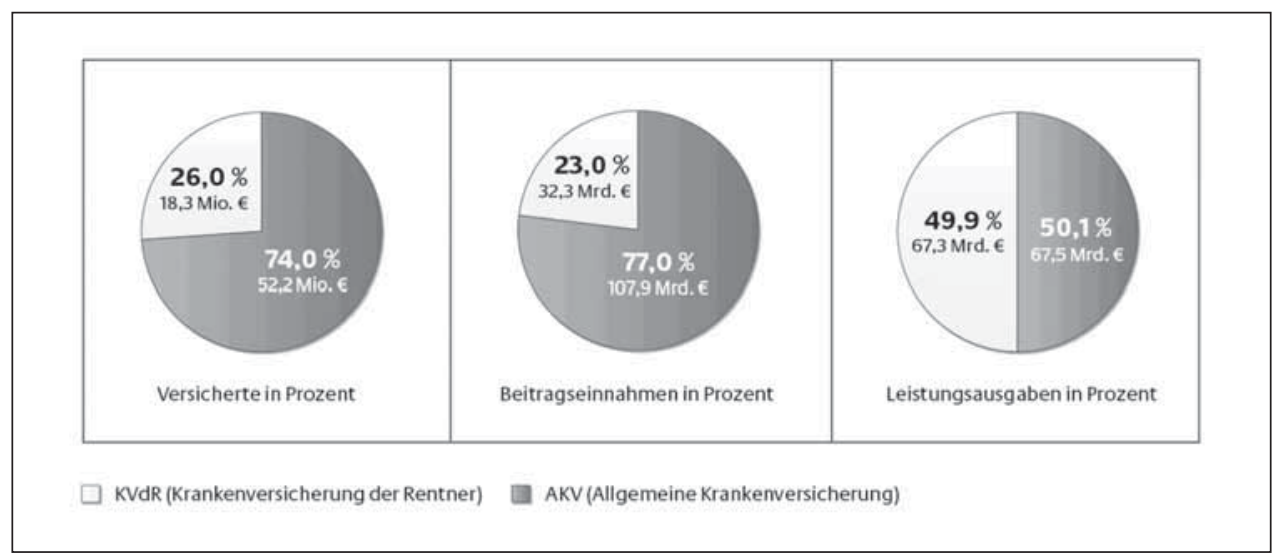

Quelle: www.die-gesundheitsreform.de (1. September 2006) 
durchschnittlich häufig unter die Regelungen zur Überschreitung der Belastungsgrenze nach $§ 62$ SGB V fallen. ${ }^{99}$

In die gleiche Richtung gehen Vorschläge, den solidarisch finanzierten Leistungsumfang der gesetzlichen Krankenversicherung einzuschränken. Auch dies hätte bei altersabhängig steigenden Gesundheitsausgaben eine transfer-mindernde Wirkung, ${ }^{100}$ da weiterhin nachgefragte, über das Leistungsangebot der gesetzlichen Krankenkassen hinausgehende Gesundheitsleistungen durch $\mathrm{Zu}$ käufe finanziert werden müssten, die nicht zu Lasten der Solidargemeinschaft gingen. In dem Maße, in dem ältere Versicherte mehr Leistungen konsumieren als jüngere, ginge diese Privatisierung der finanziellen Folgen von Krankheit ebenfalls stärker zu Lasten älterer Generationen, was zu einer generationengerechteren Verteilung der demographisch bedingten Steigerung der Gesundheitsausgaben beitragen könnte.

Der Sachverständigenrat für die Konzertierte Aktion im Gesundheitswesen hat in diesem Zusammenhang eine Art duales Versicherungssystem vorgeschlagen. ${ }^{101} \mathrm{Nach}$ diesem Konzept wird der Leistungskatalog der gesetzlichen Krankenversicherung in Grund- und Wahlleistungen aufgespaltet. ${ }^{102}$ Der solidarischen und paritätischen Finanzierung unterläge dann nur noch eine Grundversorgung. Diese könnte individuell durch Wahlleistungen ergänzt werden, wobei die hierfür erforderlichen Mittel ausschließlich durch (freiwillige und risikoäquivalente) Beiträge der Versicherten aufgebracht werden müssten. ${ }^{103}$ Verstärkt werden könnte dieser Effekt noch durch die ebenfalls vom Sachverständigenrat vorgeschlagene Möglichkeit für Versicherte, die Grundleistungen nicht nur durch Wahlleistungen zu ergänzen, sondern auch durch abwählbare Leistungen individuell einzuschränken, wenn ihr Kosten-Nutzen-Verhältnis vom Versicherten nicht als ausreichend positiv eingeschätzt wird. ${ }^{104} \mathrm{Da}$ dies natürlich mit einer entsprechenden Beitragsreduktion verbunden wäre, könnten sich jüngere Versicherte auf diese Weise auf Wunsch selbst von den finanziellen Folgen der demographischen Alterslast entlasten.

Generelle Leistungsreduktionen (und damit letztlich auch Ausweitungen von Zuzahlungen) zum Zwecke einer stärkeren Beteilung älterer Generationen an den finanziellen Folgen des demographischen Wandels sind jedoch aus systematischen Gründen abzulehnen: Während in der gesetzlichen Rentenversicherung die Transfers von den Renten wegen Erwerbsminderung und Rehabilitationsleistungen abgesehen - ausschließlich von jungen zu alten Versicherten geleistet werden, fußt das Solidarprinzip in der gesetzliche Krankenversicherung nicht in erster Linie auf der Dichotomie von Jung und Alt, sondern auf dem Gegensatz von Gesund und Krank. ${ }^{105}$ Das bedeutet, dass (zu befürwortende) Kürzungen des Rentenniveaus grundsätzlich nur ältere Menschen träfen, wohingegen Einschränkungen bei den Gesundheitsleistungen - aufgrund des sich im Alter ändernden Ausgabenprofils - zwar tendenziell, aber eben nicht ausschließlich zu höheren Belastungen Älterer führten: Ebenso betroffen wären junge Versicherte, die wegen einer (ins- besondere chronischen) Erkrankung einen Leistungsbedarf haben, der ansonsten nur dem Durchschnitt wesentlich älterer Kohorten entspricht; nicht in die Pflicht genommen würden umgekehrt Senioren mit einem - für ihr Alter eher untypisch - positiven Gesundheitszustand. Entsprechende Reformen würden also nicht - wie intendiert - intergenerativ, sondern interpersonell in Abhängigkeit von individuellen Krankheitsbildern wirken.

Wie in der gesetzlichen Renten- muss daher auch in der gesetzlichen Krankenversicherung ein Weg gefunden werden, der an der Dichotomie von Jung und Alt und nicht an derjenigen von Gesund und Krank ansetzt. Da der Bezug zum Leistungsrecht aus systematischen Erwägungen ausscheidet, gerät unweigerlich das Beitragsrecht ins Blickfeld. Auf den Punkt gebracht bedeutet dies, dass ältere Versicherte eine höhere - und damit in aller Regel auch risikoäquivalentere - Beitragslast tragen sollten als bisher, da sie den lange Jahrzehnte funktionierenden Generationenvertrag durch ihr verändertes generatives Verhalten nicht ausreichend erfüllt haben, was für die Finanzierung der erhöhten Gesundheitsausgaben ihrer Generation indes unabdingbar gewesen wäre. Erforderlich ist also zunächst eine adäquate Grenzziehung zwischen jüngeren und älteren Versicherten. Hier böte sich eine Differenzierung nach der sog. allgemeinen Krankenversicherung und der Krankenversicherung der Rentner (KVdR) nach § 5 Abs.1 Nr. 11 SGB V an.

Diskutiert werden können grundsätzlich eine altersbezogene Erhöhung des Beitragssatzes einerseits und die weitere Einbeziehung von zusätzlich zu Renten bezogenen Einkünften in die Beitragspflicht zur gesetzlichen Krankenversicherung andererseits. Eine entsprechende Erhöhung des Beitragssatzes nur für Rentnerinnen und Rentner würde dem Grundgedanken der solidarischen Finanzierung der gesetzlichen Krankenversicherung zuwiderlaufen, deren Prinzip es ja gerade ist, die wirtschaftliche Leistungsfähigkeit der einzelnen Mitglieder der Solidargemeinschaft dadurch zu berücksichtigen, dass als Beitrag ein einheitlicher Satz vom jeweiligen Einkommen erhoben wird. ${ }^{106}$ Was bleibt, ist mithin eine Verbreiterung der Beitragsbemessungsgrundlage.

Diesbezüglich ist es sinnvoll, sich die bestehenden unterschiedlichen Alterssicherungskonzepte zu veranschaulichen: Grundsätzlich lassen sich drei Gestaltungsformen unterscheiden, nämlich die private Altersvorsorge, die staatlich organisierte Rentenversicherung und die (ergänzende) betriebliche Alterssicherung. ${ }^{107}$ Das Konglomerat aller drei Institutionen wird in der deutschen Sozialpolitik regelmäßig mit dem Begriff der Drei-SäulenKonzeption charakterisiert. ${ }^{108}$

Der Beitragspflicht in der KVdR unterliegen - neben dem Arbeitseinkommen - zur Zeit nur die Einkünfte aus der staatlich organisierten Säule der Alterssicherung, also die Renten der gesetzlichen Rentenversicherung und die Versorgungsbezüge ( $\$ 237$ SGB V). Hier erscheint es im Hinblick auf die Drei-Säulen-Konzeption der deutschen Alterssicherung systemgerechter, neben den bislang berücksichtigungsfähigen Versorgungsbezügen (§ 229 SGB 
V) auch Einkünfte aus der privaten Altersvorsorge der Beitragsberechnung zugrunde zu legen. Da diese in $\mathrm{Zu}-$ kunft einen immer größeren Teil der Lebensgrundlage der Rentnergeneration darstellen werden, sollte sich auch die Beitragsbemessung an der wirtschaftlichen Leistungsfähigkeit insgesamt orientieren. ${ }^{109}$ Hinsichtlich des Beitragssatzes könnte sich der Gesetzgeber an § 248 SGB V orientieren; die Beiträge würden dann in Höhe des halben allgemeinen Beitragssatzes der zuständigen Krankenkasse erhoben und von den Rentnerinnen und Rentnern alleine getragen.

\section{Resümee}

Der Umstand, dass die deutsche Bevölkerung zahlenmäßig schrumpft und zugleich altert, hat unabweisbare und bereits jetzt absehbare Auswirkungen auf die sozialen Sicherungssysteme der Bundesrepublik Deutschland. In der gesetzlichen Rentenversicherung kann der Beitragssatz bei ungehinderter Entwicklung bis zum Jahr 2040 auf über 30\% der Bruttolöhne steigen. Eine Umstellung vom derzeitigen Umlageverfahren auf ein Kapitaldeckungsverfahren ist kein Allheilmittel: Die angesammelten Vermögenswerte stoßen wegen des Bevölkerungsrückgangs auf eine entsprechend reduzierte Nachfrage; und einer abrupten Umstellung steht die Rechtsprechung des Bundesverfassungsgerichts entgegen, wonach bestimmte rentenversicherungsrechtliche Rechtspositionen den Eigentumsschutz des Art. 14 GG genießen. Als Alternative kommt neben einer Verlängerung der Lebensarbeitszeit eine schrittweise Absenkung des Rentenniveaus in Betracht. Letztere ist durch private Vorsorge zu kompensieren.

Auch das Umlageverfahren in der gesetzlichen Krankenversicherung basiert auf der Prämisse einer „gesunden“ Altersstruktur der Bevölkerung. Kennzeichnend in der Krankenversicherung ist jedoch nicht primär die Dichotomie von Alt und Jung, sondern von Gesund und Krank. Generelle Leistungsreduktionen stoßen auf systematische Grenzen, ebenso wie eine Erhöhung des Beitragssatzes nur für Rentenbezieher. Stattdessen könnte die Bemessungsgrundlage für die Beiträge in der gesetzlichen Krankenversicherung verbreitert werden.

Für alle Systeme gilt gleichermaßen, dass nicht nur die Bevölkerung, sondern auch die Politik aus dem „demographischen Schlummer" erwacht.

\section{Literatur}

Bäcker, Gerhard et al.: Sozialpolitik und soziale Lage in Deutschland. Band 2. Gesundheit und Gesundheitssystem, Familie, Alter, Soziale Dienste, Wiesbaden 2000.

Baltes, Paul B.: Über die Zukunft des Alterns. Hoffnung mit Trauerflor, in: Baltes, Margret und Montada, Leo (Hrsg.): Produktives Leben im Alter, Frankfurt am Main und New York 1996, 29-68.

Bingler, Klaus und Bosbach, Gerd: Kein Anlass zu Furcht und Panik. Fakten und Mythen zur „demographischen Katastrophe“, in: Deutsche Rentenversicherung 2004, 725-749.

Birg, Herwig: Die demographische Zeitenwende. Der Bevölkerungswandel in Deutschland und Europa, München 2001.
Boeck, Jürgen et al.: Sozialpolitik in Deutschland. Eine systematische Einführung, Wiesbaden 2004.

Börsch-Supan, Axel H.: Das deutsche Rentenversicherungssystem: Probleme und Perspektiven, in: Wille, Eberhard (Hrsg.): Entwicklung und Perspektiven der Sozialversicherung, Baden-Baden 1999, 21-67.

Börsch-Supan, Axel: Übergangsmodelle vom Umlage- zum Kapitaldeckungsverfahren in der deutschen Rentenversicherung, in: Seel, Barbara (Hrsg.): Sicherungssysteme in einer alternden Gesellschaft. Perspektiven sozialer Sicherung zwischen Selbstverantwortung und Solidarität, Frankfurt am Main und New York 1998, 198-213.

Breyer, Friedrich und Ulrich, Volker: Demographischer Wandel, medizinischer Fortschritt und der Anstieg der Gesundheitsausgaben, in: DIW-Wochenbericht 2000, 374-379.

Breyer, Friedrich: Individuelle und kollektive Sicherungsversprechen im demographischen Wandel, in: Seel, Barbara (Hrsg.): Sicherungssysteme in einer alternden Gesellschaft. Perspektiven sozialer Sicherung zwischen Selbstverantwortung und Solidarität, Frankfurt am Main und New York 1998, 48-76.

Bundesministerium für Familie und Senioren (Hrsg): Erster Altenbericht Die Lebenssituation älterer Menschen in Deutschland, Bonn 1993.

Bundesministerium für Gesundheit und Soziale Sicherung (Hrsg.): Nachhaltigkeit in der Finanzierung der Sozialen Sicherungssysteme. Bericht der Kommission, Berlin 2003.

Butzer, Hermann: Fremdlasten in der Sozialversicherung, Tübingen 2001. CDU Bundesvorstand (Hrsg.): Zur Zukunft der sozialen Sicherungssysteme. Bericht der Kommission „Soziale Sicherheit“, Eigendruck 2003.

Deutsche Rentenversicherung Bund (Hrsg.): Rentenversicherung in Zeitreihen, Berlin 2005.

Deutsche Rentenversicherung Bund (Hrsg.): Von Ansparphase bis Zulage - „Riestern“ leicht gemacht, Berlin 2006.

Deutscher Bundestag: Endbericht der Enquête-Kommission „Demographischer Wandel - Herausforderungen unserer älter werdenden Gesellschaft an den Einzelnen und die Politik“, veröffentlicht als Bundestags-Drucksache 14/8800 (2002).

Deutscher Bundestag: Endbericht der Enquête-Kommission „Strukturreform der gesetzlichen Krankenversicherung", veröffentlicht als Bundestags-Drucksache 11/6380 (1990).

Ebsen, Ingwer: Verfassungs- und europarechtliche Vorgaben, in: Schulin, Bertram (Hg.), Handbuch des Sozialversicherungsrechts. Band 3. Rentenversicherungsrecht, München 1999, 133-163.

Ehrentraut, Oliver und Raffelhüschen, Bernd: Rente mit 67 - ein unvermeidbarer Schritt, in: Wirtschaftsdienst 2003, 627-630.

Erbsland, Manfred et al.: Die Auswirkungen der Bevölkerungsstruktur auf Ausgaben und Beitragssatz der gesetzlichen Krankenversicherung, in: Wille, Eberhard (Hrsg.): Entwicklung und Perspektiven der Sozialversicherung, Baden-Baden 1999, 173-196.

Fenge, Robert: Pareto-Efficiency of the Pay-As-You Go Pension System With Intergenerational Fairness, in: Finanzarchiv 1995, 357-363.

Fries, James F.: Aging, natural death, and the compression of morbidity, in: The New England Journal of Medicine 1980, 130-135.

Geißler, Rainer und Meyer, Thomas: Struktur und Entwicklung der Bevölkerung, in: Geißler, Rainer: Die Sozialstruktur Deutschlands. Zur gesellschaftlichen Entwicklung mit einer Zwischenbilanz zur Vereinigung, Opladen ${ }^{2}$ 1996, 333-357.

Gleitze, Wilfried: Kommentierung von § 28e SGB IV, in: Gleitze, Wilfried et al.: Gemeinschaftskommentar zum Sozialgesetzbuch Gemeinsame Vorschriften für die Sozialversicherung, Neuwied und Frankfurt am Main 1992, 394-396.

Göhde, Thomas et al.: Gesetz zur Sicherung der nachhaltigen Finanzierungsgrundlagen der gesetzlichen Rentenversicherung (RV-Nachhaltigkeitsgesetz), in: Mitteilungen der LVA Rheinprovinz 2004, 289-317.

Grohmann, Heinz: Alterssicherung im Wechsel der Generationen, in: Deutsche Rentenversicherung 2004, 185-199.

Herden, Rose-Elisabeth und Münz, Rainer: Bevölkerung, in: Schäfers, Bernhard und Zapf, Wolfgang (Hrsg.): Handwörterbuch zur Gesellschaft Deutschlands, Opladen² 2001, 75-88.

Kaltenbach, Helmut: Die Rentenversicherung im Konzept der „Drei Säulen“, in: Ruland, Franz (Hrsg.): Handbuch der gesetzlichen Rentenversicherung. Festschrift aus Anlaß des 100-jährigen Bestehens der gesetzlichen Rentenversicherung, Neuwied und Frankfurt am Main 1990, 425-450.

Kaufmann, Franz-Xaver: Schrumpfende Gesellschaft. Vom Bevölkerungsrückgang und seinen Folgen, Frankfurt am Main 2005.

Kostorz, Peter: „Später (k)eine Rente(?)“. Anmerkungen zur Demografiefalle und zur Rentenkrise, in: Die Rentenversicherung 2004, 61-68.

Kostorz, Peter: Die Verweisung im Recht der Sozialversicherung. Eine Untersuchung zum „Recht auf Faulheit“ in der gesetzlichen Kranken-, Renten- und Unfallversicherung, Baden-Baden 2002.

Kostorz, Peter: Versicherungsfremde Leistungen in der Gesetzlichen Krankenversicherung. Eine sozialpolitische Analyse unter besonderer 
Berücksichtigung der historischen Entwicklung des Leistungskatalogs der GKV, Frankfurt am Main et al. 1998.

Langen, Hans-Georg: Die neue Rentenanpassung ab 1.7.2001, in: Die Angestelltenversicherung 2001, 239-243.

Lenze, Anne: Rentenreform zwischen Eigentumsschutz und Gleichheitssatz. Zugleich eine Besprechung der BSG-Entscheidung vom 31.7.2002 zur Frage des Eigentumsschutzes der Rentenanpassung, in: Neue Zeitschrift für Sozialrecht 2003, 505-513.

Mackenroth, Gerhard: Die Reform der Sozialpolitik durch einen deutschen Sozialplan, in: Boettcher, Erik (Hg.): Sozialpolitik und Sozialreform. Ein einführendes Lehr- und Handbuch der Sozialpolitik, Tübingen 1957, 43-74.

Meyer, Wolfgang: Rechtliche Grundstrukturen der gesetzlichen Altersrentenversicherung, in: Die Rentenversicherung 2003, 21-26.

Minn, Norbert: Melde- und Einzugswesen, in: Schulin, Bertram (Hrsg.), Handbuch des Sozialversicherungsrechts. Band 1. Krankenversicherungsrecht, München 1994, 1232-1288.

Montgomery, Frank Ulrich: Krankenversicherung - Quo vadis?, unter: www.montgomery.de/vereinte (23. August 2001).

Naegele, Gerhard und Kauss, Thomas: Demographie und Sozialepidemiologie - Zur These vom demographisch bedingten Anstieg der Gesundheitsausgaben, in: Igl, Gerhard und Naegele, Gerhard (Hrsg.): Perspektiven eine sozialstaatlichen Umverteilung im Gesundheitswesen, München 1999, 63-85.

Neumann, Lothar F. und Schaper, Klaus: Die Sozialordnung der Bundesrepublik Deutschland, Frankfurt am Main und New York ${ }^{4} 1998$.

Oberdieck, Veit: Beitragssatzexplosion in der gesetzlichen Krankenversicherung? Demographische und medizintechnische Determinanten der Beitragssatzdynamik und ihre reformpolitischen Implikationen, Duisburg 1998.

Ohsmann, Sabine und Stolz, Ulrich: Entwicklung der Rendite in der gesetzlichen Rentenversicherung. Betrachtungen zur Rendite der aktuellen und künftigen Altersrenten, in: Die Angestelltenversicherung 2004, 56-62.

Papier, Hans-Jürgen: Der Einfluss des Verfassungsrechts auf das Sozialrecht, in: von Maydell, Bernd Baron und Ruland, Franz (Hrsg.): Sozialrechtshandbuch (SRH), Baden-Baden ${ }^{3}$ 2003, 81-139.

Pareto, Vilfredo: Manuale di economia politica, Mailand 1906.

Pfeiffer, Doris und Walzik, Eva: Demographischer Wandel und Kostenentwicklung im Gesundheitswesen. Sozialer Sprengstoff oder ein Effekt unter vielen, in: Die Ersatzkasse 1997, 309-316.

Reinhard, Hans-Joachim: Demographischer Wandel und Alterssicherung in Deutschland, in: Reinhard, Hans-Joachim (Hrsg.): Demographischer Wandel und Alterssicherung. Rentenpolitik in neun europäischen Ländern und den USA im Vergleich, Baden-Baden 2001, 15-56.

Ruland, Franz und von der Heide, Dirk: Rente mit 67 ohne Alternative, in: Wirtschaftsdienst 2003, 633-636.

Ruland, Franz: Der neue Nachhaltigkeitsfaktor in der Rentenanpassungsformel und seine Auswirkungen, in: Die Sozialgerichtsbarkeit 2004, 327-334.

Ruland, Franz: Rentenversicherung, in: von Maydell, Bernd Baron und Ruland, Franz (Hrsg.): Sozialrechtshandbuch (SRH), Baden-Baden 2003, 958-1060.

Sachverständigenrat für die Konzertierte Aktion im Gesundheitswesen: Sondergutachten 1995. Gesundheitsversorgung und Krankenversicherung 2000. Mehr Ergebnisorientierung, mehr Qualität und meh Wirtschaftlichkeit, Baden-Baden 1995.

Samuelsen, Paul A. und Nordhaus, William D.: Volkswirtschaftslehre. Grundlagen der Makro- und Mikrotheorie. Band 2, Köln ${ }^{8} 1987$.

Schäfers, Bernhard: Gesellschaftlicher Wandel in Deutschland. Ein Studienbuch zur Sozialstruktur und Sozialgeschichte der Bundesrepublik, Stuttgart ${ }^{6} 1995$

Schäffle, Albert E. F.: Der korporative Hülfskassenzwang, Tübingen² 1884 .

Schaper, Klaus: Sicherung im Pflegefall und Generationenvertrag, in: Zeitschrift für Sozialreform 1993, 87-102.

Schmähl, Winfried: Perspektiven der Alterssicherung - Anmerkungen zu Konzeptionen und Instrumenten, in: Seel, Barbara (Hrsg.) Sicherungssysteme in einer alternden Gesellschaft. Perspektiven sozialer Sicherung zwischen Selbstverantwortung und Solidarität, Frankfurt am Main und New York 1998, 154-177.

Schmalor, Michael: Der Gesamtsozialversicherungsbeitrag, Sankt Augustin ${ }^{6} 1999$.

Schmid, Josef et al.: Sozialprognose. Die Belastung der nachwachsenden Generation, München 2000.

Schnapp, Friedrich E. und Kostorz, Peter: Demographische Entwicklung, soziale Sicherungssysteme und Zuwanderung, in: Zeitschrift für Ausländerrecht und Ausländerpolitik 2002, 163-171.

Schnapp, Friedrich E.: Die arbeitsrechtliche Fürsorgepflicht - Legitimation für den Arbeitgeberanteil in der gesetzlichen Rentenversicherung?, in: Söllner, Alfred et al. (Hrsg.): Gedächtnisschrift für Meinhard Heinze, München 2005, 815-829.

Schnapp, Friedrich E.: Eigentumsschutz, in: Landesversicherungsanstalt Rheinland-Pfalz (Hrsg.): Zukunft der Rente - Rente der Zukunft. 1. Sozialrechtstagung am 18./19. März 2004 in Speyer, Speyer 2004, 47-69.

Schnapp, Friedrich E.: Sozialstaatlichkeit im Spannungsfeld von Eigenverantwortung und Fürsorge, in: Deutsches Verwaltungsblatt 2004, 1053-1061.

Schreiber, Wilfried: Existenzsicherheit in der industriellen Gesellschaft. Vorschläge zur „Sozialreform“, Köln 1955.

Seidler, Andreas et al.: Auswirkungen einer weiteren Steigerung der Lebenserwartung auf den medizinischen Versorgungsbedarf, in: Die Ersatzkasse 1996, 317-322.

Sinn, Hans-Werner: The Crisis of Germany's Pension Insurance System and How It Can Be Resolved, München 1999.

Statistisches Bundesamt (Hrsg.): Bevölkerung Deutschlands bis 2050. 10. koordinierte Bevölkerungsvorausberechnung, Wiesbaden 2003.

Steffen, Johannes: Die Anpassung der Renten in der Rentenversicherung der Arbeiter und Angestellten von der Rentenreform 1957 bis zum AVmEG, [Manuskript] Bremen 2002.

Ullrich, Carsten G.: Solidarität im Sozialversicherungsstaat. Die Akzeptanz des Solidarprinzips in der gesetzlichen Krankenkasse, Frankfurt am Main und New York 2000.

Verbrugge, Lois: Longer Life But Worsening Health? Trends in Health and Mortality of Middle-aged and Older Persons, in: Milbank Memoria Fund Quarterly 1984, 475-519.

von Nell-Breuning, Oswald: Die Produktivitätsrente, in: Zeitschrift für Sozialreform 1956, 97-101.

Wallerath, Maximilian: Rentenversicherung und Verfassungsrecht, in: Ruland, Franz (Hg.): Handbuch der gesetzlichen Rentenversicherung. Festschrift aus Anlaß des 100jährigen Bestehens der gesetzlichen Rentenversicherung, Neuwied und Frankfurt am Main 1990, 281-323.

Wenner, Ulrich: Rentenniveau und Grundgesetz, in: von Wulffen, Matthias und Krasney, Otto Ernst (Hrsg.): Festschrift 50 Jahre Bundessozialgericht, Köln et al. 2004, 625-643.

Wiegard, Wolfgang und Bach, Stefan: Finanzwissenschaft, in: Zimmermann, Klaus F. (Hrsg.): Neue Entwicklungen in der Wirtschaftswissenschaft, Heidelberg 2002, 43-112

Zweifel, Peter: Bevölkerung und Gesundheitswesen: Ein SisyphusSyndrom?, in: Felderer, Bernhard (Hg.): Bevölkerung und Wirtschaft, Berlin 1990, 373-386.

\section{Fußnote}

1 Diese Erkenntnis ist nicht neu; vgl. bereits Schäffle 1884, 16 der die "Voraussetzung, daß der gesetzliche Zwangsverband stets die nöthige Zahl zahlungsfähiger Mitglieder" hat, als einzige Bedingung für eine funktionierende Sozialversicherung beschreibt. Dabei geht er indes davon aus, ein „nationales System von Versicherungskorporationen“ könne „ja nicht ganz und rasch aussterben“.

2 Dem demographischen Wandel demgegenüber mit weitaus weniger Skepsis begegnend Bingler/Bosbach 2004, die ihrem Beitrag resümierend vorwegschicken, langfristige Projektionen der Bevölkerungsentwicklung könnten aufgrund zahlreicher Unwägbarkeiten höchstens einen hypothetischen Wert beanspruchen, so dass sich die „Situation - selbst unter der Voraussetzung, dass die veröffentlichten Berechnungen zutreffend wären - längst nicht so dramatisch dar[stelle], wie vielfach behauptet wird" und "dass ein erheblicher Teil der Finanzierungsprobleme für die sozialen Sicherungssysteme nicht der demographischen Entwicklung, sondern den veränderten ökonomischen Rahmenbedingungen geschuldet" sei (a.a.O., 725).

3 Vgl. etwa Geißler/Meyer 1996, 333 sowie Schäfers 1995, 88.

4 Anders Herden/Münz 2001, 75, nach denen diese Wandlungsprozesse bereits von der Bedeutung des Begriffs Bevölkerung umfasst werden.

5 Geißler/Meyer 1996, 344.

7 Vgl. auch Geißler/Meyer 1996, 344.

8 Vgl. Geißler/Meyer 1996, $339 \mathrm{f}$.

9 Die Geburtenrate in Deutschland gehört damit zu einer der niedrigsten weltweit. Niedrigere Geburtenziffern weisen in der Europäischen Union nur noch Spanien und Italien (etwa 1,2) sowie Griechenland und Österreich (etwa 1,3) auf; vgl. Statistisches Bundesamt 2003, 12.

11 Ebenfalls kritisch Schäfers 1995, 89 sowie Geißler/Meyer 1996, 336 f. 12 Vgl. Birg 2001, 47 und öfters.

13 Dieser Ansatz ist insbesondere deshalb interessant, weil er unterschiedliche Ansätze zur Erklärung des immensen Geburtenrück- 
gangs integriert, so dass mit seiner Hilfe empirische Befunde erklärt werden können, die mit den bisher diskutierten und eher isoliert betrachteten Ursachen - wie etwa die Rationalisierung de Familienplanung durch Mittel zur Empfängnisverhütung, die Emanzipation und sog. Enthäuslichung der Frau, ein anspruchsvollerer Lebensstil potentieller Eltern oder die zunehmende gesellschaftliche Akzeptanz von Kinderlosigkeit (hierzu ausführlicher Geißler/Meyer 1996, 340 f.) - kaum zu fassen waren, auch wenn diese stets als sich wechselseitig beeinflussende Aspekte eines komplexen Bedingungsgefüges angesehen wurden. So lässt sich beispielsweise allein mit Birgs Theorie der biographischen Opportunitätskosten schlüssig erklären, warum die sinkende durchschnittliche Nettoreproduktionsrate nicht - wie regelmäßig vereinfachend dargestellt wird - auf dem Trend zur Ein-Kind-Familie beruht, sondern auf der Tatsache, dass etwa ein Drittel aller Frauen zeitlebens kinderlos bleiben, während die Geburtenrate innerhalb der Gruppe von Frauen, die sich bereits für die Gründung einer Familie entschieden haben, weiterhin den nahezu idealen Wert von durchschnittlich rund zwei Kindern pro Frau hat (a.a.O., 73 ff.): Nach der Geburt des ersten Kindes haben sich die Eltern bereits langfristig gebunden, so dass ein zweites oder drittes Kind keine weitere generelle Beschneidung biographischer Alternativen bedeutet. Damit zusammen hängt ein Phänomen, das Birg sehr plastisch mit dem Begriff des „demographisch-ökonomischen Paradoxons" umschreibt (a.a.O., 42 ff.): Die durchschnittliche Anzahl der Geburten pro Frau sinkt trotz steigenden Realeinkommens, d.h. die Menschen leisten sich um so weniger Kinder, je mehr sie es sich aufgrund ihrer Einkommenssituation eigentlich könnten. Den Grund hierfür erkennt Birg darin, dass eine positive wirtschaftliche Entwicklung sowohl einer Volkswirtschaft als auch ihrer einzelnen Haushalte ja gerade auf der beschriebenen Flexibilität und Mobilität der Arbeitnehmer(innen) basiert, so dass wachsender ökonomischer Erfolg zwangsläufig mit einer Erhöhung der Zahl kinderloser Frauen, also einer gesamtgesellschaftlichen Abnahme der durchschnittlichen Nettoproduktionsrate korreliert.

14 Ebenso Statistisches Bundesamt 2003, 10 ff., das eine Erhöhung der Geburtenziffern nur für das Gebiet der neuen Länder auf das Niveau im früheren Bundesgebiet prognostiziert.

15 Vgl. Geißler/Meyer 1996, 344.

16 Vgl. Statistisches Bundesamt 2003, 14 ff. Für das Jahr 2050 wird sogar von einer Lebenserwartung von bis zu 82,6 bzw. 88,1 Jahren ausgegangen.

17 Vgl. Naegele/Kauss 1999, 66.

18 Die Prognose des Statistischen Bundesamtes 2003, 16 geht für das Jahr 2035 von einer fernen Lebenserwartung eines 60-jährigen von 22,7 Jahren bzw. 27,1 Jahren einer gleichaltrigen Frau aus; für das Jahr 2050 wird ein Anstieg auf 23,7 bzw. 28,2 Jahre erwartet - das sind 3,6 bzw. 4,1 Jahre mehr als heute (im früheren Bundesgebiet).

20 Vgl. Naegele/Kauss 1999, 65.

21 Die Grenze zwischen Erwerbs- und Rentenalter wird in den bislang vorliegenden Studien mithin nicht immer einheitlich gezogen. Aus rein sozialrechtlicher Sicht ist prima vista auf die Grenze von 65 Jahren abzustellen, da ein Anspruch auf eine Regelaltersrente grundsätzlich erst mit der Vollendung des 65. Lebensjahres besteht, § 35 Nr. 1 SGB VI. Empirisch betrachtet ist der Altenquotient ${ }_{60}$ jedoch erheblich aussagekräftiger, da in den vergangenen Jahren das durchschnittliche Rentenzugangsalter - bezogen auf alle Versichertenrenten (Renten wegen Alters gem. \$ 33 Abs. 2 SGB VI sowie Renten wegen verminderter Erwerbsfähigkeit gem. § 33 Abs. 3 SGB VI) - konstant bei unter 61 Jahren lag; vgl. Deutsche Rentenversicherung Bund 2005, 111.

23 Vgl. Statistisches Bundesamt 2003, 32.

25 Die gängige Redeweise von den versicherten Beschäftigten als Beitragszahlern, die hier der Einfachheit halber beibehalten wird, blendet den Umstand aus, dass alleiniger Beitragszahler und -schuldner der Arbeitgeber ist. Er refinanziert sich in Höhe des hälftigen Beitrags beim Arbeitnehmer. Das ist ganz einhellige Meinung; siehe z.B. BSG SozR 3-2400 § 25 Nr. 6, 27; BSGE 64, 110, 113; BSGE 48, 195, 197; s.a. BGHZ 133, 370, 375; BFHE 172, 467, 470; Gleitze 1992, Rdnr. 1; Minn 1994, Rdnr. 181; Schmalor 1999, 410.

26 Vgl. auch Schaper 1993, 87, der den Vertrag wie folgt skizziert: „Die jeweils aktive Generation in der Generationenfolge ... leistet an die ältere Generation (do) und fordert und erhält als Gegenleistung (ut des) Rentenzahlungen der nachfolgenden Generation im nächsten Zeitstadium." Dem bestehenden kollektiven und öffentlichen Rentensystem liegt mithin ein Zweigenerationenvertrag zwischen der jeweiligen aktiven Generation einerseits und der betreffenden Rentnergeneration andererseits zugrunde.

27 So auch Börsch-Supan 1999, 36.

28 Vgl. hierzu umfassend Schmid et al. 2000, 162.

30 Vgl. etwa Börsch-Supan 1998, 199.

31 Vgl. etwa Schmähl 1998, 161 f. sowie Kostorz 2004, 63 f.
32 Vgl. hierzu auch Breyer 1998, 51.

33 Hierzu ausführlicher Schnapp/Kostorz 2002, 166; zur Renditeentwicklung in der gesetzlichen Rentenversicherung vgl. generell Ohsmann/Stolz 2004, zu deren verfassungsrechtlichen Implikationen Papier 2003, Rdnr. 56.

34 Mackenroth 1957, 45 (Hervorhebung im Original; Erstabdruck in: Schriften des Vereins für Socialpolitik NF, Band 4, Berlin 1952).

35 So beispielsweise Reinhard 2001, 29; ähnlich Boeckh et al. 2004, 108 ff. sowie Kostorz 2004, 64.

36 BVerfGE 53, 257, 289, fortgeführt bis BVerfGE 100, 1.

37 BVerfGE 69, 272, 300.

38 So etwa Ebsen 1999, Rdnr. 59 oder Wallerath 1990, Rdnr. 85 ff.; vgl. hierzu auch Meyer 2003, 25 sowie Schnapp 2004, 49 ff. und 67 ff.

39 Zur Rentenberechnung nach der geltenden Rentenformel vgl. Schnapp 2004, $1058 \mathrm{ff}$. sowie Schnapp 2005, $824 \mathrm{ff}$.

40 So auch Ebsen 1999, Rdnr. 64.

41 Dieses Problem der Doppelbelastung hat die Ökonomie lange Jahre beschäftigt und beschäftigt sie wohl auch heute noch. Gesucht wurde und wird ein Weg, der doch einen sog. pareto-effizienten Übergang vom Umlage- zum Kapitaldeckungsverfahren möglich macht. Der italienische Soziologe und Nationalökonom Vilfredo Pareto [1906] ging davon aus, dass praktische Wirtschaftspolitik dann optimal sei, wenn der Nutzen mindestens eines Individuums gesteigert werden könne, ohne gleichzeitig den Nutzen mindestens eines anderen zu reduzieren (vgl. etwa Samuelson/Nordhaus 1987, 440) - oder m.a.W: Möglichst vielen soll es besser gehen, ohne dass es nur einem schlechter geht. Ein solcher pareto-optimaler Übergang vom Umlage- zum Kapitaldeckungsverfahren gilt in der Wirtschaftswissenschaft als de facto ausgeschlossen; vgl. etwa Wiegard/Bach 2002, 70 oder Fenge 1995.

42 Vgl. zum folgenden Sinn 1999, 15 f. sowie rezipierend Wiegard/Bach 2002, $72 \mathrm{f}$.

43 Sinn 1999, 16.

44 Vgl. Wiegard/Bach 2002, 72.

46 Vgl. oben Anm. ${ }^{26}$.

47 Ideengeschichtlich geht dies auf Schreiber 1955 zurück, der sich für zwei Zweigenerationenverträge zwischen der heranwachsenden und der aktiven Generation einerseits und der aktiven und der älteren Generation andererseits aussprach; vgl. hierzu auch Kaufmann 2005, $204 \mathrm{ff}$.

48 Vgl. von Nell-Breuning 1956, 98 „Die produktive Generation, die ihn [den sog. neuen Plan, also die Umstellung der Rentenversicherung auf das Umlageverfahren; d.V.] eineinführt [sic!], übernimmt eine zusätzliche Leistung zugunsten der ihr vorangegangenen Generation, indem sie sich bereit erklärt, dieser Leistungen zukommen zu lassen, die nicht bzw. nur zu einem geringen Teil durch entsprechende Vorleistungen (Beitragszahlungen in entsprechender Höhe) verdient sind. Sie verläßt sich darauf, was sie jetzt für die ihr voraufgegangene Generation aufwendet, das werde die ihr nachfolgende Generation ihr entgelten. ... Der Schlüssel zur Altersversorgung welcher Generation auch immer liegt nicht in der Geldrechnung, auch nicht in einer güterwirtschaftlichen Produktions- oder Produktivitätsrechnung; er liegt in der biologischen Struktur des Volkskörpers."

50 Vgl. auch Neumann/Schaper 1998, 180.

51 Pointierend bereits Kostorz 2004, 66: „Für »halbe Generationen « können als Gegenleistung keine »vollen Renten « erwartet werden. “ Ebenso Grohmann 2004, 194: Es ist die „mittlere Generation ..., deren Geburtenhäufigkeit die absehbare massive Alterung der Gesellschaft letztlich bewirkt hat. Sie wird es freilich auch sein, die die Folgen in ihrer eigenen Alterssicherung zu tragen hat."

52 Mit Neumann/Schaper 1998, 180 ist jedoch zu betonen, dass es hier in keiner Weise um „persönliche Schuld“, sondern lediglich um empirisch objektivierbare gesellschaftliche Wandlungsprozesse geht.

53 Davon unabhängig spricht sich unter anderem Schmähl 1998, 166 dafür aus, die gesetzliche Rentenversicherung soweit wie möglich auf ihre Kernaufgaben zu beschränken, und zwar auf die einkommensäquivalente Absicherung im Alter und bei Invalidität. „Maßnahmen der Arbeitsmarkt- und Beschäftigungspolitik, der Familienpolitik und der gezielten Armutsvermeidung sollten den dafür inhaltlich zuständigen Institutionen zugeordnet werden."

54 Betroffen waren in erster Linie die Altersrenten für Arbeitslose, für schwerbehinderte Menschen, für Frauen und für langjährig Versicherte; vgl. hierzu ausführlicher Ruland 2003, Rdnr. $136 \mathrm{ff}$.

55 Auch nach Anhebung der Altersgrenzen können die Renten ab dem 60. bzw. 63. Lebensjahr in Anspruch genommen werden, allerdings unter Inkaufnahme von Rentenminderungen. Sie haben den Zweck, die längere Rentenlaufzeit auszugleichen und betragen 0,3\% der Rente für jeden Monat, den die Rente vorzeitig bezogen wird. Diese Abschläge sollen versicherungsmathematisch so berechnet sein, dass eine Frühverrentung keine negativen finanziellen Auswirkungen für die gesetzliche Rentenversicherung mehr haben soll; teilweise wird 
der Abschlagssatz von 3,6\% p.a. allerdings als zu niedrig angesetzt erachtet; vgl. etwa Schmähl 1998, 175.

56 Hierzu ausführlich Kostorz 2002, 35 ff.

57 Vgl. Deutsche Rentenversicherung Bund 2005, 134.

$58 \mathrm{Vgl}$. bereits oben Anm. ${ }^{18}$.

59 So auch Deutscher Bundestag 2002, 159

60 So bereits Kostorz 2004, 65.

61 Vgl. Bundesministerium für Gesundheit und Soziale Sicherung 2003, 82 ff. bzw. CDU Bundesvorstand 2003, 42. Siehe hierzu auch Ehrentraut/Raffelhüschen 2003 sowie Ruland/von der Heide 2003.

62 So auch Kostorz 2004, 65.

63 Vgl. hierzu ausführlicher Lenze 2003 unter Bezugnahme auf BSGE 90, 11 sowie Wenner 2004.

64 Mit der modifizierten Bruttoanpassung folgen die Renten zwar nach wie vor den Löhnen und Gehältern, doch beeinflussen seitdem steuerliche Veränderungen die Höhe der Rentenanpassungen ebenso wie Änderungen der Beitragssätze zur Kranken-, Pflege- und Arbeitslosenversicherung nicht mehr. Vgl. hierzu Steffen 2002, 32 ff. sowie ausführlich Langen 2001.

65 Vgl. hierzu Ruland 2004 sowie Göhde et al. 2004.

66 So auch Ruland 2004, 330. Das schließt sog. Nullrunden indes nicht aus; absolute Rentenkürzungen sind durch eine Sicherungsklausel allerdings ausgeschlossen.

67 Vgl. Deutsche Rentenversicherung Bund 2005, 234.

68 Vgl. Bundesministerium für Gesundheit und Soziale Sicherung 2003, $106 \mathrm{f}$.

69 Vgl. hierzu ausführlicher Kostorz 2004, 66 f.

70 Hierzu umfassend Deutsche Rentenversicherung 2006.

71 Vgl. Bundesministerium für Gesundheit und Soziale Sicherung 2003, 108.

72 Vgl. Bundesministerium für Familie und Senioren 1993.

73 Vgl. Naegele/Kauss 1999, 66.

74 Vgl. Pfeiffer/Walzik 1997, 314.

75 Vgl. Naegele/Kauss 1999, 68.

76 Inwieweit die Praxisgebühr nach § 28 Abs. 4 SGB V diesem Phänomen entgegenwirken kann, wird sich in Zukunft noch herausstellen müssen.

77 Hierzu grundlegend Verbrugge 1984.

78 Hierzu grundlegend Fries 1980.

80 Baltes 1996, 52.

81 Vgl. Pfeiffer/Walzik 1997, 314. Zu den chronischen Krankheiten zählen insbesondere Herz-/Kreislauf- und Bronchialerkrankungen, Demenz und Diabetes mellitus. Hinsichtlich der Multimorbidität gehen Studien von etwa fünf bis neun nebeneinander existierenden Diagnosen bei den 70- bis 90jährigen aus; vgl. Naegele/Kauss 1999, 69.

82 Vgl. Oberdieck 1998, 103. Nach Naegele/Kauss 1999, 73 wird dieser Effekt zusätzlich dadurch verstärkt, dass ältere Patienten besonders ausgabenintensive Leistungen nachfragen. Es handelt sich hierbei in erster Linie um die besonders teure Krankenhausbehandlung, aber auch um die ambulante ärztliche Behandlung sowie um die Versorgung mit Arzneimitteln.

83 Naegele/Kauss 1999, 71; vgl. auch Seidler et al. 1996, 317-322.

84 Nach Naegele/Kauss 1999, 71 könnte die Manifestation der "compressed morbidity" und ihrer Folgen im Extrem womöglich sogar jenseits des biologischen Maximalalters hinausgeschoben werden. „Das Resultat wäre ein Tod, für die meisten in der neunten Lebensdekade, ohne daß man längere Zeit krank wäre. ... Wie eine nach außen noch recht ordentlich aussehende Uhr würden wir plötzlich stehenbleiben, relativ gesund sterben“ (Baltes 1996, 55).

85 Vgl. Naegele/Kauss 1999, 77.

86 Vgl. Naegele/Kauss 1999, 73.

88 Ebenso Montgomery 2001.

89 Vgl. Erbsland et al. 1999, 190. Andere Schätzungen bei Schmid et al. 2000, 167 f. Unter Berücksichtigung des medizinisch-technischen Fortschritts in bisherigem Umfang muss sogar mit einem Anstieg auf ca. $25 \%$ gerechnet werden; vgl. Breyer/Ulrich 2001 sowie Montgomery 2001. Nicht unerheblich ist in diesem Zusammenhang, dass es gerade die Kombination von Altersentwicklung und medizinischtechnischem Fortschritt ist, die sich derart dramatisch auswirkt: Die Weiterentwicklung der Medizin kommt in erster Linie alten Menschen und ihren Überlebenschancen zugute; so wurden die größten Fortschritte bislang bei der Verbesserung der Lebensqualität alter Menschen und der Verlängerung ihres Lebens erreicht; vgl. Montgomery 2001. Zu diesem in der Sozialmedizin "Sisyphus-Syndrom“ genannten Phänomen vgl. auch Zweifel 1990, 373.

91 Vgl. hierzu Deutscher Bundestag 1990, 310.

92 Vgl. hierzu die ausführliche empirische Untersuchung von Ullrich 2000, der sowohl die Solidarbereitschaft der Nettozahler als auch die „Solidaritätswürdigkeit“ der Nettoempfänger untersuchte.
93 Vgl. Ullrich 2000,139.

94 Vgl. Ullrich 2000, 121

95 Im Ergebnis wohl ebenso Ullrich 2000, 122, der davon ausgeht, dass eine solche Erwartung ,ein hinreichendes Vertrauen in die Stabilität und Zuverlässigkeit des wohlfahrtsstaatlichen Arrangements" voraussetzt, „daß die durch die aktuelle Beitragszahlung erworbenen Ansprüche (als Netto-Zahler) zu einem späteren Zeitpunkt auch tatsächlich realisiert werden können. Dies ist im Fall der Erwartungsreziprozität prekär, da die erworbenen Ansprüche bei einer neuen Gruppe zunächst nur virtueller Netto-Zahler eingelöst werden müssen. Bei diesen ist jedoch keineswegs sicher, ob sie bereit und fähig sind, ihren Teil zu diesem "Generationenvertrag « beizutragen".

96 Vgl. oben S. ${ }^{15}$ (hier!).

97 Vgl. Erbsland et al. 1999, 175.

98 Eine grundsätzlich, d.h. von der Überforderungsklausel des § 62 SGB $\mathrm{V}$ unabhängige Pflicht zur Selbstbeteiligung ergibt sich bereits jetzt aus $\S \S 61$ i.V.m. 31 Abs. 3 bzw. 39 Abs. 4 SGB V. Danach besteht eine Zuzahlungspflicht zu Arzneimitteln in Höhe von 10\% des

Abgabepreises, mindestens jedoch $5 €$ und höchstens $10 €$. Die Zuzahlung zur Krankenhausbehandlung beträgt $10 €$ für maximal 28 Tage im Kalenderjahr, also maximal $280 €$.

99 Im Ergebnis ebenso Oberdiek 1998, 158.

100 Vgl. Oberdiek 1998, 157.

101 Vgl. Sachverständigenrat für die Konzertierte Aktion im Gesundheitswesen 1995, Rdnr. $438 \mathrm{ff}$.

102 Um zu einer adäquaten Differenzierung dieser beiden Leistungsbereiche zu gelangen, wäre es erforderlich, eine Bezugsgrundlage zu definieren, die für alle Versicherten gemeinsam und einheitlich gelten müsste. Versicherten, die keine Wahlmöglichkeit in Anspruch nähmen, müsste also ein gemeinsames und einheitliches Pflichtleistungsangebot aller Krankenkassen gewährt werden; vgl. Sachverständigenrat für die Konzertierte Aktion im Gesundheitswesen 1995, Rdnr. 441. Hierzu müsste nach Auffassung des Sachverständigenrates der Leistungskatalog von sog. versicherungsfremden Leistungen bereinigt werden: „Der so bereinigte Leistungsumfang kann dann zum Ausgangspunkt für eine $\mathrm{Zu}$ - und Abwahl von Leistungen gemacht werden." $\mathrm{Zu}$ versicherungsfremden Leistungen vgl. ausführlich Butzer 2001 und Kostorz 1998.

103 Als Zuwahlleistungen böten sich neben den ausgegliederten versicherungsfremden Leistungen nach Meinung des Sachverständigenrats für die Konzertierte Aktion im Gesundheitswesen 1995, Rdnr. 439 und 446 bestimmte alternative Therapieangebote, präventive Kuren oder medizinische Maßnahmen an, bei denen (noch) Zweifel an ihrer Wirksamkeit bestehen. Darüber hinaus böten sich als mögliche Wahlleistungen ein Teil der Hilfsmittel, wie beispielsweise Hörhilfen, sowie einzelne Heilmittelbereiche, wie etwa Bäder oder Massagen an.

104 So wäre es nach dem Sachverständigenrat für die Konzertierte Aktion im Gesundheitswesen 1995, Rdnr. 440 und 450 ff. beispielsweise denkbar, dass Versicherte einen Teil des Krankheitsrisikos selbst übernehmen, indem sie die auftretenden Krankheitskosten in einem Jahr bis zu einem bestimmten Betrag selbst übernehmen. Eine weitere Möglichkeit wäre die sektorenspezifische Abwahl von Leistungen, beispielsweise bezogen auf die zahnärztliche Versorgung oder die ambulante Medikamentenversorgung.

105 Zwar wird in der Sozialpolitik (wie etwa von Bäcker et al. 2000, 63) regelmäßig vertreten, der solidarische Ausgleich beinhalte in der gesetzlichen Krankenversicherung neben der risikobezogenen Umverteilung auch einen Generationenausgleich, da die hohen Krankheitsausgaben für Rentnerinnen und Rentner nur teilweise durch eigene Beiträge gedeckt werden und daher im Erwerbsleben Stehende für diesen Personenkreis mitzahlen müssten. Zu betonen ist jedoch, dass es sich bei dem beschriebenen Generationenausgleich nur mittelbar um ein eigenständiges Solidaritätskriterium handelt und es sich hierbei aufgrund sich im Alter ändernder Krankheitsbilder vielmehr um einen Teilaspekt der risikobezogenen Umverteilung handelt.

106 So etwa Oberdieck 1998, 156, der dies - bei einer prozentualen Beitragserhebung wie in der vom Solidarprinzip geprägten gesetzlichen Krankenversicherung allerdings fälschlicherweise - damit begründet, dass „Renten ohnehin niedriger als die Arbeitseinkommen ausfallen“. Erbsland et al. 1999, 191 weisen in diesem Zusammenhang zu Recht darauf hin, dass eine Erhöhung des Beitragssatzes allein für die Rentnergeneration zu einer im Vergleich mit ihr überproportionalen Belastung der Erwerbsgeneration führen würde, da sich die Rentenkassen, die den Beitrag zur Hälfte tragen müssen (§ 249a SGB V), aus Beiträgen der Erwerbstätigengeneration finanzieren.

107 Vgl. etwa Bäcker et al. 2000, 244.

108 Vgl. hierzu ausführlich Kaltenbach 1990.

109 So sieht beispielsweise auch § 238 SGB V für freiwillig versicherte Rentner vor, dass alle Einnahmen der Beitragsbemessung zugrunde zu legen sind, „die die wirtschaftliche Leistungsfähigkeit des freiwilligen Mitglieds bestimmen“. Diese Diskrepanz zwischen der 
Beitragserhebung bei versicherungspflichtigen und freiwillig versicherten Rentnern ist nebenbei schon jetzt insofern erstaunlich und unter systematischen Gesichtspunkten fragwürdig, als die Zuordnung zu einer der beiden Personenkreise in aller Regel davon abhängt, ob der Versicherte zu seiner aktiven Zeit versicherungspflichtig oder freiwillig versichert war (vgl. die Voraussetzungen zur KVdR, also zur Rentner-Pflichtversicherung nach $\S 5$ Abs. 1 Nr. 11 SGB V). Insofern lässt sich die Frage stellen, warum Versicherte, die zu Zeiten ihrer Erwerbstätigkeit freiwillig der Solidargemeinschaft der Krankenversicherung beigetreten sind und dadurch Transfers zu älteren Generationen geleistet haben, nun insofern schlechter gestellt sind, als sie höhere Beiträge zu entrichten haben als Pflichtversicherte der gleichen Kohorte.

\section{Die Zukunft der ambulanten Patientenversorgung}

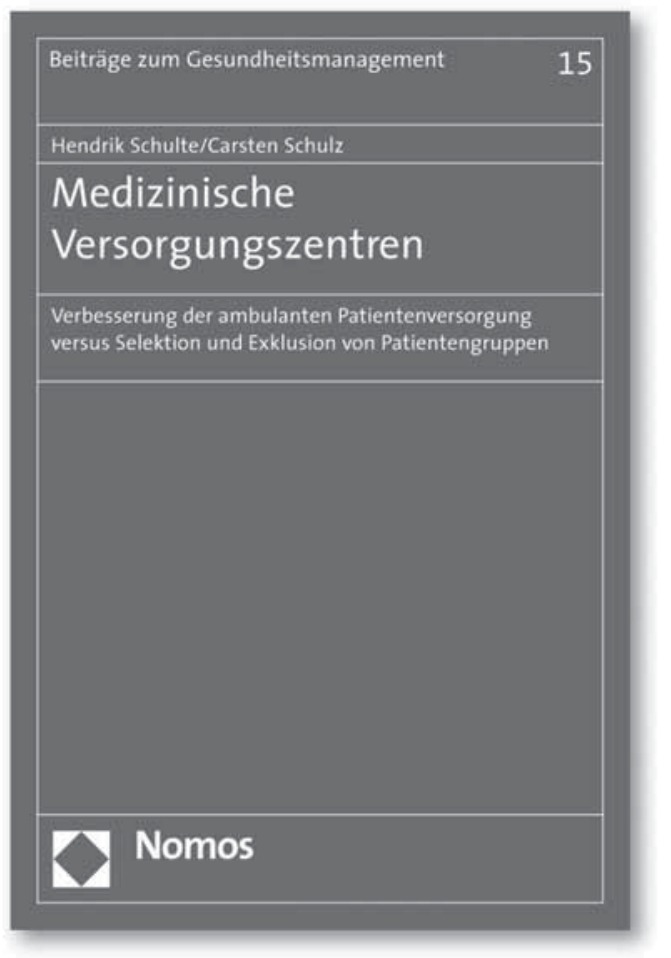

\author{
Medizinische Versorgungszentren \\ Verbesserung der ambulanten Patientenversorgung \\ versus Selektion und Exklusion von Patientengruppen \\ Von Dr. Hendrik Schulte und Dr. Carsten Schulz \\ 2006, 294 S., brosch., 49,- $€$, ISBN 3-8329-2430-2 \\ (Beiträge zum Gesundheitsmanagement, Bd. 15)
}

Das Werk untersucht, inwieweit die im Rahmen des Gesundheitsmodernisierungsgesetzes (GMG) zugelassenen Medizinischen Versorgungszentren (MVZ) die ambulante Patientenversorgung in Deutschland verbessern. Dies erfolgt auf der Basis einer in verschiedenen Einrichtungen durchgeführten Ärzte- und Patientenbefragung. Zudem wird untersucht, ob MVZ Selektions- und Exklusionstendenzen im Gesundheitswesen erhöhen oder mindern.

\section{Nomos}

Bitte bestellen Sie bei Ihrer Buchhandlung oder bei: Nomos Verlagsgesellschaft | 76520 Baden-Baden | www.nomos.de 